\title{
DIRECTIONS FOR THE LONG EXACT COHOMOLOGY SEQUENCE IN MOORE CATEGORIES
}

\author{
DIANA RODELO
}

\begin{abstract}
A new method for realizing the first and second order cohomology groups of an internal abelian group in a Barr-exact category was introduced in [6] and [10]. The main role, in each level, is played by a direction functor. This approach can be generalized to any level $n$ and produces a long exact cohomology sequence. By applying this method to Moore categories we show that they represent a good context for non-abelian cohomology, in particular the Baer Extension Theory.
\end{abstract}

\section{Introduction}

One of the goals in Homological Algebra is to form a long exact cohomology sequence of abelian groups, given a short exact sequence of modules over a ring $R$ with identity. The starting point is the well known property of the additive functor $\operatorname{Hom}_{R}(X,-): R$-Mod $\rightarrow$ Ab of preserving left exact sequences. Intuitively, the following question is raised: given a short exact sequence of $R$-modules $N \longmapsto P \rightarrow Q$, can we prolong the exact sequence of abelian groups $0 \rightarrow \operatorname{Hom}_{R}(X, N) \rightarrow \operatorname{Hom}_{R}(X, P) \rightarrow \operatorname{Hom}_{R}(X, Q)$ (infinitely) to the right?

The affirmative answer is given through equivalence classes of $n$-fold extensions equipped with the Baer sum. These abelian groups can be computed as cohomology groups and are used to construct a long exact cohomology sequence.

The problem can be generalized to the following expression: Given a short exact sequence

$$
N \stackrel{k}{\longrightarrow} P \stackrel{p}{\longrightarrow} Q
$$

in $\operatorname{Ab}(\mathcal{E})$, can the exact sequence of abelian groups

$$
0 \longrightarrow \operatorname{Hom}_{\mathcal{E}}(1, N) \stackrel{k_{*}}{\longrightarrow} \operatorname{Hom}_{\mathcal{E}}(1, P) \stackrel{p_{*}}{\longrightarrow} \operatorname{Hom}_{\mathcal{E}}(1, Q)
$$

be extended (infinitely) to the right?

Received January 3, 2007.

The author gratefully acknowledges financial support from FCT/Centre of Mathematics of the University of Coimbra. 
In the first place, due to the interest in having $\operatorname{Ab}(\mathcal{E})$ an abelian category, we establish $\mathcal{E}$ as a Barr-exact category. Moreover, based on the classical (non-abelian) cases, the answer is centered on the research of a "good" definition for the $n$-th (non-abelian) cohomology group of an internal abelian group $A$ in $\mathcal{E}, \mathbb{H}_{\mathcal{E}}^{n}(A)$. We begin with $\mathbb{H}_{\mathcal{E}}^{0}(A)=\operatorname{Hom}_{\mathcal{E}}(1, A)$.

The first step to extend (2) was made by Barr in [1]. The interpretation of $\mathbb{H}_{\mathcal{E}}^{1}(A)$ used principal group actions, also known as $A$-torsors. This approach stopped at level 1 with a 6 -term exact sequence. The first long exact cohomology sequence formed from a short exact sequence was achieved by Duskin and Glenn in [16] and [18]. Their theory was founded on simplicial methods.

Afterwards, Bourn also obtained a long exact cohomology sequence in [4] by using internal $n$-groupoids. The cohomology groups $\mathbb{H}_{\mathcal{E}}^{n}(A)$ are defined through internal $(n-1)$-groupoids for which there is an internal $n$-functor from its induced indiscrete internal $n$-groupoid to $\mathrm{K}_{n}(A)$. Besides relating $A$-torsors and internal groupoids at level 1 , this approach provided an easy generalization mechanism for higher orders.

More recently, Bourn presented a new method for realizing the first cohomology group in [6] and the second in [10]. The key in each level consists of a functor, called direction (of order 2 for level 2), which is used to obtain the first and second cohomology groups, respectively. This approach can be extended to higher levels also by means of internal $n$-groupoids [25]. The cohomology groups $\mathbb{H}_{\mathcal{E}}^{n}(A)$ are defined through internal $(n-1)$-groupoids having direction $A$. The main progress from [4] is due to the (final, discrete fibration) factorization of internal $n$-functors. This allows the description of the cohomology groups through internal $(n-1)$-groupoids with a property (of having a certain direction) rather than exhibiting extra data (the existence of a certain kind of internal $n$-functor).

In the first part of this work we briefly mention the method developed in [4] through, what we call, $A$-labeled internal $n$-groupoids as the supportive background for the direction functor approach. We recall the basics for the direction functor theory for levels 1 and 2 and give the guidelines for extending the theory to higher levels.

Moore categories where introduced by Gerstenhaber in [17]. His approach intended to represent a good context for non-abelian (co)homology, in particular for the Baer Extension Theory. The method was developed from the 
direct translation of the classical theory and became, in many aspects, extremely technical. In the second part of this work we shall see that, what we propose as, Moore categories actually represent such a good context by applying the direction functor theory to their fibres. This provides a simple description of the cohomology groups of any order. Extending classical terminology to these categories, we define the Baer sum of equivalence classes of crossed $n$-fold extensions. For the particular case of groups, the equivalent interpretations of [20] and [19] can be recovered and similar interpretations for Lie algebras can be obtained. See [11] for the application of the direction functor approach to the context of homological categories.

We would like to emphasize the fact that the initial non-abelian context used to obtain realizations of the (non-abelian) cohomology groups is misleading, since we can always consider, through the direction functors, an abelian point of view.

Special thanks to Dominique Bourn for all the suggestions made for improving this work.

\section{The starting point}

In the following sections $\mathcal{E}$ represents a Barr-exact category with finite limits. We represent the full subcategory of objects with global support, i.e. such that $!_{X}: X \rightarrow 1$ is a regular epimorphism, by $\mathcal{E}_{\#}$. We denote by $A$ an object equipped with an internal abelian group structure $\left(A, m_{A}, e_{A}, i_{A}\right)$.

Given a short exact sequence (1), we want to prolong the 3 -term exact sequence (2) to the right. Based on the classical examples, the infinite extension of (2) is focussed on obtaining suitable definitions for the $n$-th cohomology groups of an internal abelian group. The sequence formed this way is, thus, called the long exact cohomology sequence. We begin with the following definition:

Definition 1.1. The 0-th cohomology group of $A$ is $\mathbb{H}_{\mathcal{E}}^{0}(A)=\operatorname{Hom}_{\mathcal{E}}(1, A)$. This gives a 0 -th order cohomology functor $\mathbb{H}_{\mathcal{E}}^{0}: \operatorname{Ab}(\mathcal{E}) \rightarrow \mathrm{Ab}$.

The cohomology groups $\mathbb{H}_{\mathcal{E}}^{n}(A), n \geq 1$, will be defined as the abelian group of the connected components of a suitable monoidal category. Recall that two objects $X$ and $Y$ in a category $\mathcal{C}$ are connected when there exists a zig-zag between them; we write $X \sim Y$. Being connected determines an equivalence relation on the objects of $\mathcal{C}$. We call the equivalence classes the connected components of $\mathcal{C}$ and denote them by $\pi_{0}(\mathcal{C})$. 


\section{A-labeled internal $n$-groupoids}

In this section we briefly recall the interpretation of the cohomology groups $\mathbb{H}_{\mathcal{E}}^{n}(A)$ given in [4], through, what we call, $A$-labeled internal $n$-groupoids. We adopt different notations.

An internal groupoid $\underline{X}_{1}$ in $\mathcal{E}$ is represented by a diagram

$$
\underline{X}_{1}: X_{1} \underset{c}{\stackrel{d}{\stackrel{ }{\rightleftarrows}}} X_{0}
$$

equipped with a composition morphism $m: X_{1} \times_{X_{0}} X_{1} \rightarrow X_{1}$. We denote the category of internal groupoids in $\mathcal{E}$ by $\operatorname{Grd}(\mathcal{E})$. We have a forgetful functor ()$_{0}: \operatorname{Grd}(\mathcal{E}) \rightarrow \mathcal{E}$, associating to every internal groupoid $\underline{X}_{1}$ its object of 0 -cells $X_{0}$. The fibre of $\left({ }_{-}\right)_{0}$ at $X_{0}$ is represented by $\operatorname{Grd}_{X_{0}}(\mathcal{E})$. In particular, $\operatorname{Grd}_{1}(\mathcal{E}) \cong \operatorname{Gp}(\mathcal{E})$ and we denote by $\mathrm{K}_{1}(G)$ the internal groupoid associated to an internal group $G$ in $\mathcal{E}$. Moreover, $\left(_{-}\right)_{0}$ has a fully faithful right adjoint given by the indiscrete groupoid functor denoted by $\nabla_{1}$. We represent by $\underline{X}_{1}^{\bumpeq}$ the kernel pair of the terminal object $\underline{X}_{1} \rightarrow \nabla_{1}\left(X_{0}\right)$ in $\operatorname{Grd}_{X_{0}}(\mathcal{E})$.

It is easy to see that $\left(_{-}\right)_{0}$ is a fibration; the $\left(_{-}\right)_{0}$-cartesian morphisms are the fully faithful internal functors, i.e. internal functors $\underline{\varphi}_{1}: \underline{X}_{1} \rightarrow \underline{Y}_{1}$ such that

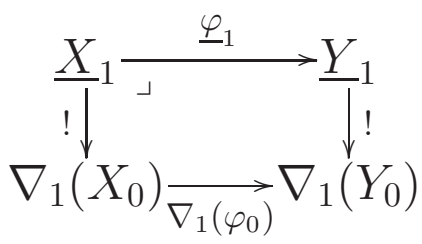

is a pullback; an internal functor is $\left({ }_{-}\right)_{0}$-invertible when it is an isomorphism on 0-cells. These two classes of morphisms form a factorization system.

Another important factorization system is given by the discrete fibrations and final internal functors. An internal functor $\underline{\varphi}_{1}: \underline{X}_{1} \rightarrow \underline{Y}_{1}$ is a discrete fibration when the diagram

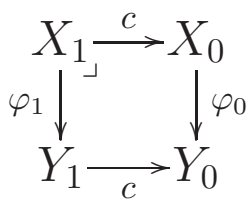

(or, equivalently, the diagram with the domains) is a pullback; it is final when it is orthogonal to any discrete fibration ([3]). 
Remark 2.1. ([3]) Any final internal functor $\underline{\chi}_{1}: \nabla_{1}(X) \rightarrow \underline{Y}_{1}$ is always indiscrete, i.e. $\underline{Y}_{1} \cong \nabla_{1}(\hat{X})$ and $\underline{\chi}_{1} \cong \nabla_{1}(x)$ for some morphism $x: X \rightarrow$ $\hat{X}$. The (final, discrete fibration) factorization of an internal functor $\underline{\varphi}_{1}$ : $\nabla_{1}(X) \rightarrow \mathrm{K}_{1}(A)$ produces a discrete fibration ${\underline{q_{\hat{X}}}}_{1}: \nabla_{1}(\hat{X}) \rightarrow \mathrm{K}_{1}(A)$, thus an isomorphism $\hat{X} \times \hat{X} \cong \hat{X} \times A$. Moreover, $\hat{X} \in \mathcal{E}_{\#}$ whenever $X \in \mathcal{E}_{\#}$.

We consider the comma category $\nabla_{1} \downarrow \mathrm{K}_{1}(A)$ for the functors $\nabla_{1}: \mathcal{E}_{\#} \rightarrow$ $\operatorname{Grd}(\mathcal{E})$, restricted to objects with global support, and $\mathrm{K}_{1}(A): \mathbb{1} \rightarrow \operatorname{Grd}(\mathcal{E})$. An object of $\nabla_{1} \downarrow \mathrm{K}_{1}(A)$ is a pair $\left(X, \underline{\varphi}_{1}: \nabla_{1}(X) \rightarrow \mathrm{K}_{1}(A)\right)$ which, by Remark 2.1, satisfies $\left(X, \underline{\varphi}_{1}\right) \sim\left(\hat{X}, \underline{q}_{\hat{X}}\right)$. The internal functor $\underline{\varphi}_{1}$ can be seen as an internal groupoid whose 0-cells are the elements of $X$ such that between each ordered pair of elements of $X$ there exists a unique 1-cell labeled by an element of $A$. This justifies the following definition:

Definition 2.2. The objects of $\nabla_{1} \downarrow \mathrm{K}_{1}(A)$ are called A-labeled internal groupoids.

For higher order cohomology groups we use higher order internal groupoids. Until further specification, we consider $n \geq 2$.

An internal $n$-groupoid $\underline{X}_{n}$ in $\mathcal{E}$ is represented by a diagram

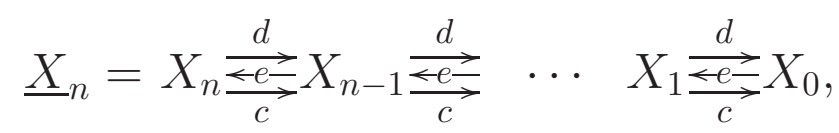

equipped with composition morphisms for all levels. We denote the category of internal $n$-groupoids in $\mathcal{E}$ by $n$ - $\operatorname{Grd}(\mathcal{E})$. For $\underline{X}_{k} \in k-\operatorname{Grd}(\mathcal{E}), n-1 \leq k \leq 0$, we represent by $n$ - $\operatorname{Grd}_{\underline{X}_{k}}(\mathcal{E})$ the category of internal $n$-groupoids over $\underline{X}_{k}$ (where $\underline{X}_{0}$ means an object of $\mathcal{E}$ ). Representing the internal $(n-1)$-groupoid of $n$-cells by $\int \underline{X}_{n}=X_{n} \underset{\rightarrow}{\leftrightarrows} X_{n-2} \underset{\rightarrow}{\rightleftarrows} \cdots X_{0}$ and the internal $(n-1)$-groupoid that cancels level $n$ by $\underline{X}_{n-1}$, we see that an internal $n$-groupoid is just an internal groupoid $\int \underline{X}_{n} \underset{\rightarrow}{\rightrightarrows} \underline{X}_{n-1}$ in $(n-1)-\operatorname{Grd}_{\underline{X}_{n-2}}(\mathcal{E})$. We have a forgetful functor ()$_{n-1}: n-\operatorname{Grd}(\mathcal{E}) \rightarrow(n-1)-\operatorname{Grd}(\mathcal{E})$, associating to every internal $n$ groupoid $\underline{X}_{n}$ the internal $(n-1)$-groupoid $\underline{X}_{n-1}$. It has a fully faithful right adjoint denoted by $\nabla_{n}$ and defined by $\nabla_{n}\left(\underline{X}_{n-1}\right)=X_{n-1}^{\bumpeq} \underset{\leftrightarrows}{\leftrightarrows} X_{n-1} \underset{\rightarrow}{\rightrightarrows} \cdots X_{0}$, where $X_{n-1}^{\bumpeq}$ is the object of parallel $(n-1)$-cells (take $\left.X_{0}^{\bumpeq}=X_{0} \times X_{0}\right)$. We 
represent by $\underline{X}_{n}^{\bumpeq}$ the kernel pair of the terminal morphism $\underline{X}_{n} \rightarrow \nabla_{n}\left(\underline{X}_{n-1}\right)$ in $n$-Grd $\underline{X}_{n-1}(\mathcal{E})$. Moreover, every internal abelian group $A$ induces an internal $n$-groupoid denoted by $\mathrm{K}_{n}(A)=A \underset{\rightarrow}{\stackrel{\rightleftarrows}{\leftrightarrows}} \underset{\rightarrow}{\rightleftarrows} \cdots 1$.

Definition 2.3. We call an internal groupoid $\underline{X}_{1}$ aspherical when $\underline{X}_{1} \rightarrow$ $\nabla_{1}\left(X_{0}\right)$ and $X_{0} \rightarrow 1$ are regular epimorphisms. An internal $n$-groupoid $\underline{X}_{n}$ is called aspherical when $\underline{X}_{n} \rightarrow \nabla_{n}\left(\underline{X}_{n-1}\right)$ is a regular epimorphism and $\underline{X}_{n-1}$ is aspherical.

We denote by $(n-) \operatorname{Asp}(\mathcal{E})$ the category of aspherical internal $(n$-)groupoids in $\mathcal{E}$.

The forgetful functor $\left({ }_{-}\right)_{n-1}$ is also a fibration and the classes of $\left({ }_{-}\right)_{n-1^{-}}$ cartesian morphisms and ( -$)_{n-1}$-invertible morphisms form a factorization system. The notions of final functors and discrete fibrations are extendable to $n-\operatorname{Grd}(\mathcal{E})$ and still form a factorization system.

Remark 2.4. ([3]) Any final internal $n$-functor $\underline{\chi}_{n}: \nabla_{n}\left(\underline{X}_{n-1}\right) \rightarrow \underline{Y}_{n}$ is always indiscrete and $\left(_{-}\right)_{n-2}$-invertible, i.e. $\underline{Y}_{n} \cong \nabla_{n}\left(\underline{\hat{X}}_{n-1}\right)$ and $\underline{\chi}_{n} \cong \nabla_{n}\left(\underline{x}_{n-1}\right)$ for some $(-)_{n-2}$-invertible internal $(n-1)$-functor $\underline{x}_{n-1}: \underline{X}_{n-1} \rightarrow \underline{X}_{n-1}$. The (final, discrete fibration) factorization of an internal $n$-functor $\underline{\varphi}_{n}$ : $\nabla_{n}\left(\underline{X}_{n-1}\right) \rightarrow \mathrm{K}_{n}(A)$ produces a discrete fibration ${\underline{q_{\hat{X}}-1}}_{n}: \nabla_{n}\left(\underline{\hat{X}}_{n-1}\right) \rightarrow$ $\mathrm{K}_{n}(A)$, thus an isomorphism $\hat{X}_{n-1}^{\bumpeq} \cong X_{n-1} \times A$. Moreover, $\underline{X}_{n-1} \in(n-1)$ $\operatorname{Asp}(\mathcal{E})$ whenever $\underline{X}_{n-1} \in(n-1)-\operatorname{Asp}(\mathcal{E})$.

We consider the comma category $\nabla_{n} \downarrow \mathrm{K}_{n}(A)$ for the functors $\nabla_{n}:(n-1)$ $\operatorname{Asp}(\mathcal{E}) \rightarrow n-\operatorname{Grd}(\mathcal{E})$, restricted to aspherical internal $(n-1)$-groupoids, and $\mathrm{K}_{n}(A): \mathbb{1} \rightarrow n-\operatorname{Grd}(\mathcal{E})$. As before, an object of $\nabla_{n} \downarrow \mathrm{K}_{n}(A)$ is a pair $\left(\underline{X}_{n-1}, \underline{\varphi}_{n}: \nabla_{n}\left(\underline{X}_{n-1}\right) \rightarrow \mathrm{K}_{n}(A)\right)$ which, by Remark 2.4 , satisfies $\left(\underline{X}_{n-1}, \underline{\varphi}_{n}\right)$ $\sim\left(\underline{\hat{X}}_{n-1},{\underline{q_{\underline{\hat{X}}} n}}_{n}\right)$. The internal $n$-functor $\underline{\varphi}_{n}$ can be seen as an internal $n$-groupoid whose 0 -cells, $\cdots,(n-1)$-cells are those of $\underline{X}_{n-1}$ such that between each ordered pair of parallel $(n-1)$-cells of $\underline{X}_{n-1}$ there exists a unique $n$-cell labeled by an element of $A$. This justifies the following definition:

Definition 2.5. The objects of $\nabla_{n} \downarrow \mathrm{K}_{n}(A)$ are called A-labeled internal n-groupoids. 
Theorem 2.6. ([4]) For $n \geq 1, \nabla_{n} \downarrow \mathrm{K}_{n}(A)$ is a monoidal symmetric category with unit $\left(\nabla_{n-1}(1), \underline{e}_{n}: \nabla_{n}(1) \rightarrow \mathrm{K}_{n}(A)\right)$ and such that $\left(\underline{X}_{n-1}, \underline{\varphi}_{n}\right) \otimes$ $\left(\underline{Y}_{n-1}, \underline{\psi}_{n}\right)=\left(\underline{X}_{n-1} \times \underline{Y}_{n-1}, \underline{\varphi}_{n} \otimes \underline{\psi}_{n}\right)$ for $\underline{\varphi}_{n} \otimes \underline{\psi}_{n} \cong \mathrm{K}_{n}\left(m_{A}\right) \cdot \underline{\varphi}_{n} \times \underline{\psi}_{n}$. Moreover, we have $\left(\underline{X}_{n-1}, \underline{\varphi}_{n}\right) \otimes\left(\underline{X}_{n-1}, \mathrm{~K}_{n}\left(i_{A}\right) \cdot \underline{\varphi}_{n}\right) \sim\left(\nabla_{n-1}(1), \underline{e}_{A}\right)$, for any object $\left(\underline{X}_{n-1}, \underline{\varphi}_{n}\right)$ in $\nabla_{n} \downarrow \mathrm{K}_{n}(A)$. As a consequence, $\pi_{0}\left(\nabla_{n} \downarrow \mathrm{K}_{n}(A)\right) \in$ $\mathrm{Ab}$.

Definition 2.7. For $n \geq 1$, the $n$-th cohomology group of $A$ is defined by $\mathbb{H}_{\mathcal{E}}^{n}(A)=\pi_{0}\left(\nabla_{n} \downarrow \mathrm{K}_{n}(A)\right)$. This gives an $n$-th order cohomology functor $\mathbb{H}_{\mathcal{E}}^{n}: \operatorname{Ab}(\mathcal{E}) \rightarrow \mathrm{Ab}$.

Theorem 2.8. ([4]) Given a short exact sequence (1), we obtain a long exact cohomology sequence

$$
\begin{gathered}
0 \longrightarrow \mathbb{H}_{\mathcal{E}}^{0}(N) \stackrel{k_{*}}{\longrightarrow} \mathbb{H}_{\mathcal{E}}^{0}(P) \stackrel{p_{*}}{\longrightarrow} \mathbb{H}_{\mathcal{E}}^{0}(Q) \stackrel{\delta^{0}}{\longrightarrow} \mathbb{H}_{\mathcal{E}}^{1}(N) \longrightarrow \cdots \\
\cdots \longrightarrow \mathbb{H}_{\mathcal{E}}^{n}(N) \stackrel{k_{*}^{n}}{\longrightarrow} \mathbb{H}_{\mathcal{E}}^{n}(P) \stackrel{p_{*}^{n}}{\longrightarrow} \mathbb{H}_{\mathcal{E}}^{n}(Q) \stackrel{\delta^{n}}{\longrightarrow} \mathbb{H}_{\mathcal{E}}^{n+1}(N) \longrightarrow \cdots,
\end{gathered}
$$

where, for simplification, we write $k_{*}^{n}$ and $p_{*}^{n}$ instead of $\mathbb{H}_{\mathcal{E}}^{n}(k)$ and $\mathbb{H}_{\mathcal{E}}^{n}(p)$, respectively.

\section{The direction functor approach}

In this section we briefly recall the direction functor theory for levels 1 and 2. The main developments of this approach can be found in [6] and [10]. The definition of the second order direction functor is based on that of the first order direction functor. So, by induction, we can generalize the notion of direction functor to any order $n, n \geq 3$. We finish by establishing the link with the theory developed in Section 2.

The details of the generalization of the direction theory can be found in [25]. In [15] the direction functor approach is completely developed for the simpler context of naturally Maltsev categories where internal groupoids are just reflexive graphs.

Whenever it is possible, we apply the Yoneda immersion in order to use elements and simplify some of the statements made below.

\subsection{The direction functor $d$.}


Definition 3.1. A ternary operation $p_{X}: X \times X \times X \rightarrow X$ is called a Maltsev operation if $p_{X}(x, x, y)=y$ and $p_{X}(x, y, y)=x$, for any elements of $X ; X$ is also called an abelian object. A Maltsev operation $p_{X}$ is said to be autonomous when it commutes with itself.

We represent by $\operatorname{AutM}(\mathcal{E})$ the category of autonomous Maltsev operations in $\mathcal{E}$. Note that, in the presence of a zero object, abelian objects are those equipped with an internal abelian group structure. The category $\operatorname{AutM}\left(\mathcal{E}_{\#}\right)$ is Barr-exact with finite products, naturally Maltsev and essentially affine (Proposition 8 of [6]).

We denote the projections of a product by $p_{X}: X \times Y \rightarrow X, \cdots$ or $p_{0}, p_{1}$ : $X \times X \rightarrow X, \cdots$ and the diagonal morphism by $s_{0}=<1,1>$.

Definition 3.2. Let $\left(X, p_{X}\right) \in \operatorname{AutM}(\mathcal{E})$. The Chasles relation associated to $p_{X}$ is the equivalence relation $C h\left[p_{X}\right]$ on $X \times X$ given by

$$
X \times X \times X \underset{p_{1,2}}{\stackrel{<p_{0}, p_{X}>}{\longrightarrow}} X \times X
$$

Definition 3.3. The direction $d(X)$ of $\left(X, p_{X}\right) \in \operatorname{AutM}\left(\mathcal{E}_{\#}\right)$ is given by the quociente of $X \times X$ by $C h\left[p_{X}\right]$.

Note that by applying the Barr-Kock Theorem to the commutative diagram

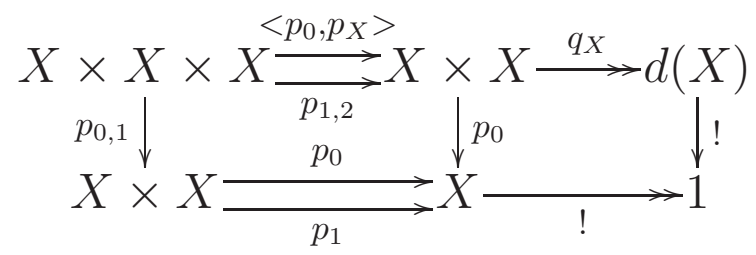

we see that the right square is a pullback. Moreover, $d(X) \in \operatorname{Ab}(\mathcal{E})$ since $X \in \mathcal{E}_{\#}$. This leads to a simpler definition:

Definition 3.4. The direction $d(X)$ of $X \in \operatorname{AutM}\left(\mathcal{E}_{\#}\right)$ is determined by the internal abelian group $d(X)=A$ making the downward diagram

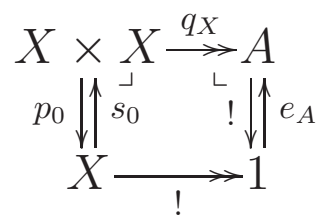

a pullback in $\operatorname{AutM}\left(\mathcal{E}_{\#}\right)$ or, equivalently, the upward diagram a pushout in $\operatorname{AutM}\left(\mathcal{E}_{\#}\right)\left(\operatorname{AutM}\left(\mathcal{E}_{\#}\right)\right.$ is essentially affine). This produces the direction functor $d: \operatorname{AutM}\left(\mathcal{E}_{\#}\right) \rightarrow \operatorname{Ab}(\mathcal{E})$. 
Remark 3.5. Properties of $d([6])$.

1: $d$ is exact and conservative.

2: $d$ is a pseudo-cofibration and every morphism in $\operatorname{AutM}\left(\mathcal{E}_{\#}\right)$ is $d$ cocartesian.

3: The fibres of $d$ are symmetric monoidal closed groupoids and the change of base functors are strongly monoidal. The tensor product of $X, Y \in d^{-1}(A)$ is defined by the codomain of the $d$-cocartesian morphism $\bar{\mu}_{X, Y}: X \times Y \rightarrow X \otimes_{1} Y$ above $m_{A}$; the unit is $A$.

4: The monoidal structure of $d^{-1}(A)$ induces an abelian group structure on its connected components.

3.2. The second order direction functor $\boldsymbol{d}_{2}$. The second order direction functor is based on the first order direction functor. We can consider $d$ for the Barr-exact category with finite limits $\operatorname{Grd}_{X_{0}}(\mathcal{E})$, denoted by $d_{X_{0}}$ : $\operatorname{AutM}\left(\left(\operatorname{Grd}_{X_{0}}(\mathcal{E})\right)_{\#}\right) \rightarrow \operatorname{Ab}\left(\operatorname{Grd}_{X_{0}}(\mathcal{E})\right)$.

Definition 3.6. An internal groupoid $\underline{X}_{1}$ is called abelian when it is an abelian object of $\operatorname{Grd}_{X_{0}}(\mathcal{E})$, i.e. $\underline{X}_{1} \in \operatorname{AutM}\left(\operatorname{Grd}_{X_{0}}(\mathcal{E})\right)$.

The category of aspherical abelian internal groupoids in $\mathcal{E}$ is denoted by $\operatorname{Asp}_{a b}(\mathcal{E})$.

As in level 1, we are interested in having a functor defined into $\operatorname{Ab}(\mathcal{E})$, the starting category for the short exact sequence (1). This is possible when $X_{0} \in \mathcal{E}_{\#}$, due to the equivalence $\operatorname{Ab}\left(\operatorname{Grd}_{X_{0}}(\mathcal{E})\right) \cong \operatorname{Ab}(\mathcal{E})$ (Theorem 9 in [10]) and determines an isomorphism $d_{X_{0}}\left(\underline{X}_{1}\right) \cong \nabla_{1}\left(X_{0}\right) \times \mathrm{K}_{1}(A)$, for some $A \in \operatorname{Ab}(\mathcal{E})$. We obtain the following construction:

Definition 3.7. The second order direction of $\underline{X}_{1} \in \operatorname{Asp}_{a b}(\mathcal{E})$ is given by the internal abelian group $d_{2}\left(\underline{X}_{1}\right)=A$ making the downward diagram

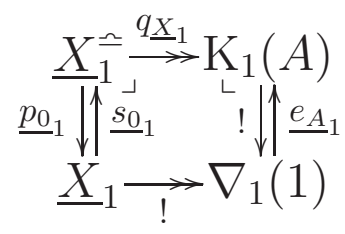

a pullback in $\operatorname{Asp}_{a b}(\mathcal{E})$ or, equivalently, the upward diagram a pushout in $\operatorname{Asp}_{a b}(\mathcal{E})$ (Proposition 1 in $[10]$ ). This produces the second order direction functor $d_{2}: \operatorname{Asp}_{a b}(\mathcal{E}) \rightarrow \operatorname{Ab}(\mathcal{E})$.

Remark 3.8. Properties of $d_{2}([10])$. 
1: $d_{2}\left(\mathrm{~K}_{1}(A)\right) \cong A$ for $q_{\mathrm{K}_{1}(A)}=\mathrm{K}_{1}\left(\backslash_{A}\right)$, for the division $\backslash_{A}=m_{A} \cdot\left(1_{A} \times\right.$ $\left.i_{A}\right)$.

2: An internal functor is $d_{2}$-invertible if and only if it is $\left({ }_{-}\right)_{0}$-cartesian.

3: $d_{2}$ preserves finite products and all existing pullbacks.

4: $d_{2}$ is a pseudo-cofibration.

5: An internal functor is $d_{2}$-cocartesian if and only if it is $(-)_{0}$-invertible; thus $d_{2}$-cocartesian morphisms are stable for finite products.

6: A $\left({ }_{-}\right)_{0}$-invertible and $d_{2}$-invertible internal functor is an isomorphism.

7: The change of base functors with respect to $(-)_{0}$ reflect isomorphisms.

8: The fibers of $d_{2}$ are symmetric monoidal categories and the change of base functors, with respect to $d_{2}$, are strongly monoidal. The tensor product of $\underline{X}_{1}, \underline{Y}_{1} \in d_{2}^{-1}(A)$ is defined by the codomain of the $d_{2^{-}}$ cocartesian morphism $\bar{\mu}_{\underline{X}_{1}, \underline{Y}_{1}}: \underline{X}_{1} \times \underline{Y}_{1} \rightarrow \underline{X}_{1} \otimes_{2} \underline{Y}_{1}$ above $m_{A}$; the unit is $\mathrm{K}_{1}(A)$.

9: Given $\underline{X}_{1}, \underline{Y}_{1} \in d_{2}^{-1}(A)$, let $\underline{X}_{1} \backslash_{2} \underline{Y}_{1}$ denote the codomain of the $d_{2^{-}}$ cocartesian map above the division $\backslash_{A}$. For each object $\underline{X}_{1} \in d_{2}^{-1}(A)$, we have $\underline{X}_{1} \backslash_{2} \mathrm{~K}_{1}(A) \in d_{2}^{-1}(A)$ such that $\underline{X}_{1} \otimes_{2}\left(\underline{X}_{1} \backslash{ }_{2} \mathrm{~K}_{1}(A)\right) \sim \mathrm{K}_{1}(A)$. As a consequence, the monoidal structure of $d_{2}^{-1}(A)$ induces an abelian group structure on its connected components.

3.3. The direction functor $d_{n}, n \geq 3$. In this section we will generalize the considerations made in Subsection 3.2 to any order $n \geq 3$. If we call objects (with global support) by (aspherical) internal 0-groupoids (so $X_{0}=$ $\underline{X}_{0}$ and $\left.A=\mathrm{K}_{0}(A)\right)$ and internal groupoids by internal 1-groupoids, then in the generalization process we should replace 0 by $n-2,1$ by $n-1$ and 2 by $n$. For higher levels, we actually have a simplification since all internal $n$-groupoids are necessarily abelian for $n \geq 2$.

From now on let $n \geq 3$ and suppose there exists an $(n-1)$-th order direction functor $d_{n-1}:(n-2)-\operatorname{Asp}_{a b}(\mathcal{E}) \rightarrow \mathrm{Ab}(\mathcal{E})$. We can consider it for the Barrexact category with finite limits $\operatorname{Grd}_{X_{0}}(\mathcal{E})$, denoted by $d_{n-1, X_{0}}:(n-2)$ $\operatorname{Asp}_{a b}(\mathcal{E}) \rightarrow \operatorname{Ab}\left(\operatorname{Grd}_{X_{0}}(\mathcal{E})\right)$. Since $X_{0} \in \mathcal{E}_{\#}$, we can use the equivalence $\mathrm{Ab}\left(\operatorname{Grd}_{X_{0}}(\mathcal{E})\right) \cong \mathrm{Ab}(\mathcal{E})$ (Theorem 9 in [10]) to determine an isomorphism $d_{n-1, X_{0}}\left(\underline{X}_{n-1}\right) \cong \nabla_{1}\left(X_{0}\right) \times \mathrm{K}_{1}(A)$, for some $A \in \operatorname{Ab}(\mathcal{E})$. We obtain the following construction: 
Definition 3.9. The $n$-th order direction of $\underline{X}_{n-1} \in(n-1)-\operatorname{Asp}(\mathcal{E})$ is given by the internal abelian group $d_{n}\left(\underline{X}_{n-1}\right)=A$ making the downward diagram

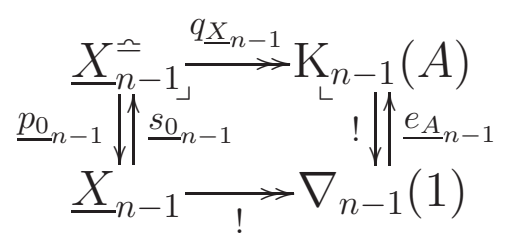

a pullback in $(n-1)-\operatorname{Asp}(\mathcal{E})$ or, equivalently, the upward diagram a pushout in $(n-1)-\operatorname{Asp}(\mathcal{E})$ (Proposition 1 in [10]). This produces the $n$-th order direction functor $d_{n}:(n-1)-\operatorname{Asp}(\mathcal{E}) \rightarrow \operatorname{Ab}(\mathcal{E})$.

Using induction and adequate adjustments for higher order internal $n$-groupoids, we can prove that $d_{n}, n \geq 3$, has the same properties as those of $d_{2}$ listed in Remark 3.8.

We have the following situation

$$
\operatorname{Ab}(\mathcal{E}) \stackrel{d_{n}}{\longleftarrow}(n-1)-\operatorname{Asp}_{a b}(\mathcal{E}) \stackrel{(-)_{n-2}}{\longrightarrow}(n-2)-\operatorname{Asp}(\mathcal{E}),
$$

with $d_{n}$ a pseudo-cofibration and $\left({ }_{-}\right)_{n-2}$ a fibration; it is a bifibration since $(-)_{n-2}$-cartesian and $d_{n}$-cocartesian morphisms commute.

3.4. The link with $A$-labeled internal $n$-groupoids. The link between the direction functor approach and the one using $A$-labeled internal $n$-groupoids, for $n \geq 1$, of Section 2 is a consequence of the (final,discrete fibration) factorization of internal $n$-functors. As mentioned in Remark 2.4, every $A$-labeled internal $n$-groupoid $\left(\underline{X}_{n-1}, \underline{\varphi}_{n}\right)$ is connected to one of the type $\left(\underline{\hat{X}}_{n-1}, \underline{q_{\hat{X}_{n-1}}}\right)$. Since ${\underline{q_{\underline{\hat{X}}}^{n-1}}}_{n}$ is a discrete fibration, then we have $d_{n}\left(\underline{\hat{X}}_{n-1}\right)=A$ (Definition 3.9).

Proposition 3.10. ([25]) There is an adjunction $\nabla_{n} \downarrow \mathrm{K}_{n}(A) \rightleftarrows d_{n}^{-1}(A)$ such that the left adjoint preserves the tensor product (up to isomorphism).

As a consequence, we have $\pi_{0}\left(\nabla_{n} \downarrow \mathrm{K}_{n}(A)\right) \cong \pi_{0}\left(d_{n}^{-1}(A)\right)$ so we could also consider

$$
\mathbb{H}_{\mathcal{E}}^{n}(A) \cong \pi_{0}\left(d_{n}^{-1}(A)\right), n \geq 1
$$

to produce a long exact cohomology sequence as (3). 


\section{Moore categories}

The notion of a Moore category introduced in [17] by Gerstenhaber consists of a long list of axioms whose independency was unclear. A comparative analysis between old notions and more recent ones made in [24] (similar to the one done in [21] for semi-abelian categories) translated them in Barr-exact ([1]) and strongly protomodular categories ([5], [8] and [7]) with zero object and cokernels. If we consider all finite colimits, we are before semi-abelian categories (which are also strongly protomodular). Due to the growing research of these categories, we will join this extra condition and propose a definition which is slightly different from the original one.

\subsection{Basic definitions.}

Definition 4.1. ([5]) A category $\mathcal{C}$ with pullbacks is called protomodular if the change of base functor, with respect to the fibration of pointed objects $\Pi: \mathrm{PtC} \rightarrow \mathcal{C}$ which associates to each split epimorphism its codomain, reflect isomorphisms.

Definition 4.2. ([1]) A category is called regular when it has kernel pairs and their coequalizers and the pullback of a regular epimorphism along any morphism exists and is a regular epimorphism. It is called Barr-exact when it is regular and every equivalence relation is effective, i.e. is a kernel pair.

Remark 4.3. It is well known that any protomodular category is always Maltsev. So we can use all the properties of regular Maltsev categories (see [2], and the references therein) in our context. In particular, we use the fact that:

1: Every Maltsev operation is necessarily autonomous.

2: The notions of internal groupoid and multiplicative reflexive graph (i.e. a reflexive graph with a composition that admits left and right units) both coincide and every reflexive graph admits at most one internal groupoid structure. Moreover, the forgetful functor from the category of internal groupoids to reflexive graphs is full.

Protomodular categories satisfy many typical group properties, so Grp is the principal example. One of these properties is the (Split) Short Five Lemma, that also characterizes the (regular) protomodular categories with zero object. If we also consider Barr-exact categories we get more group features like, for instance, the notion of semi-direct products (Definition 4.13). 
Definition 4.4. ([21]) A Barr-exact and protomodular category with zero object and finite colimits is called semi-abelian.

The notion of a normal subobject can be generalized.

Definition 4.5. ([8]) Let $\mathcal{C}$ be a category with finite limits. A monomorphism $f: X \rightarrow Y$ is said normal to an equivalence relation $R$ on $Y$ (written $f \dashv R)$ when there exists a morphism $\nabla_{X} \rightarrow R$ which is a discrete fibration.

In a protomodular category with finite limits, if a monomorphism is normal to an equivalence relation, then the relation is unique up to isomorphism. With this result, normality is no longer necessarily associated to an equivalence relation.

Definition 4.6. ([7]) A category $\mathcal{C}$ with finite limits is said strongly protomodular if the change of base functors, with respect to the fibration of pointed objects $\Pi: \mathrm{PtC} \rightarrow \mathcal{C}$, reflect isomorphisms and normal monomorphisms.

The definition of a Moore category that we propose is:

Definition 4.7. A Moore category is Barr-exact and strongly protomodular with zero object and finite colimits.

Examples 4.8. ([21, 24]) As examples of Moore categories we have: Grp, Rg and Lie (the category of Lie algebras), all abelian categories, the category of Heyting semi-lattices, $\operatorname{Grp}(\mathcal{C})$ and $\operatorname{Rng}(\mathcal{C})$ for a topos $\mathrm{C}$ with natural number object and also PtC $[X]$ for any Barr-exact and strongly protomodular category $\mathcal{C}$ with pushouts.

In the following sections $\mathcal{S}$ represents a semi-abelian category, $\mathcal{M}$ represents a Moore category and $Z$ a fixed object of $\mathcal{M}$.

4.2. Kernels and cokernels. Although the notions of normal monomorphism (with respect to an equivalence relation) and kernel are distinct, in general, the two concepts coincide in any Moore category $\mathcal{M}$. On the other hand, the notions of extremal epimorphism, regular epimorphism and strong epimorphism are equivalent, since $\mathcal{M}$ is regular. They also coincide with that of a cokernel due to the protomodularity of $\mathcal{M}$. The pullback of a regular epimorphism is a regular epimorphism because $\mathcal{M}$ is regular and any pullback of this form is always a pushout because $\mathcal{M}$ is protomodular (Proposition 14 and Corollary of [5]). 
We denote the kernel of a morphism $g: W \rightarrow X_{0}$ by an arrow $\operatorname{ker}(g)$ : $\mathrm{k}(W) \longmapsto W$. We denote cokernels by $\rightarrow$. Split epimorphism are special types of cokernels and are represented by a pair of maps $\underset{s}{\stackrel{f}{\rightleftarrows}}$, where $f \cdot s=1$.

4.3. ( $n$-fold) Extensions. A short exact sequence $\mathrm{E}: A \longmapsto X \rightarrow Z$ in $\mathcal{M}$ is called an extension in $\mathcal{M}$ of $A$ by $Z$. It is said to be singular when $A \in \operatorname{Ab}(\mathcal{M})$. We denote by $\operatorname{Sing}_{Z}(\mathcal{M})$ the category of singular extensions in $\mathcal{M}$ over $Z$. The terminal object of $\operatorname{Sing}_{Z}(\mathcal{M})$ is $Z: 1 \longmapsto Z=Z$. We denote the product of $\mathrm{E}, \mathrm{E}^{\prime} \in \operatorname{Sing}_{Z}(\mathcal{M})$ by $\mathrm{E} \times{ }_{Z} \mathrm{E}^{\prime}: A \times A^{\prime} \longmapsto X \times_{Z} X^{\prime} \rightarrow Z$.

Remark 4.9. The following hold for any commutative diagram of extensions:

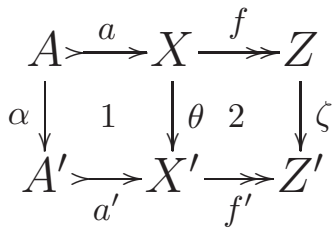

1: If $\zeta$ is a monomorphism, then 1 is a pullback.

2: ([9]) When $\zeta$ is an isomorphism, $\alpha$ is a regular epimorphism if and only if $\theta$ is a regular epimorphism.

3: ([21]) If 2 is a pushout and $\theta$ and $\zeta$ are regular epimorphisms, then $\alpha$ is a regular epimorphism.

Proposition 4.10. ([2]) Let $\mathcal{C}$ be a strongly protomodular category with zero object. The abelian objects in the slice category $\mathcal{C} / Z$ are the morphisms with an abelian kernel.

As a consequence, we have an equivalence (recall Remark 4.3.1.)

$$
\operatorname{AutM}\left((\mathcal{M} / Z)_{\#}\right) \cong \operatorname{Sing}_{Z}(\mathcal{M}) \text {. }
$$

A short exact sequence $\mathrm{E}: A \longmapsto X \rightleftarrows Z$ in $\mathcal{M}$ with section $s$, is called a split extension. When $A \in \mathrm{Ab}(\mathcal{M})$, we say that the split extension (or just $A$ ) is a $Z$-module in $\mathcal{M}$. We represent by $\operatorname{Mod}_{Z}(\mathcal{M})$ the category of $Z$-modules in $\mathcal{M}$.

Proposition 4.11. ([7]) Let $\mathcal{C}$ be a strongly protomodular category with zero object. The objects in $\mathrm{Ab}(\mathcal{C} / Z)$ are the split epimorphisms with an abelian kernel.

As a consequence, we have an equivalence

$$
\operatorname{Ab}(\mathcal{M} / Z) \cong \operatorname{Mod}_{Z}(\mathcal{M})
$$


Let $\mathrm{E}: A \longmapsto X \rightarrow Z$ be an extension in $\mathcal{M}$ and consider a monomorphism $\zeta: Z^{\prime} \longmapsto Z$ and a regular epimorphism $\alpha: A \rightarrow A^{\prime \prime}$. The composition of $\mathrm{E}$ with $\zeta$, denoted by $\mathrm{E} \zeta$, is defined from the pullback of $\zeta$ along $\mathrm{E}$; it is always an extension in $\mathcal{M}$. The composition of $\alpha$ with $\mathrm{E}$, denoted by $\alpha \mathrm{E}$, is obtained from the pushout of $\alpha$ along $\mathrm{E}$; in all the cases we consider it is an extension in $\mathcal{M}$ :
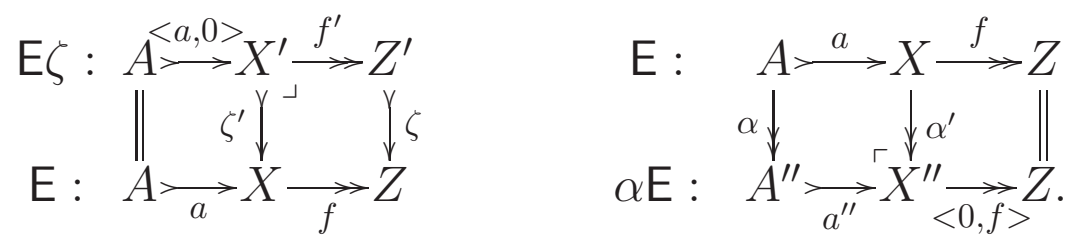

An exact sequence $\mathrm{E}: A \longmapsto X_{n-1} \rightarrow \cdots X_{1} \rightarrow X_{0} \rightarrow Z$ in $\mathcal{M}$ is called an $n$-fold extension in $\mathcal{M}$ of $A$ by $Z$. Being exact, the $n$-fold extension $\mathrm{E}$ is decomposed into $n$ extensions $\mathrm{C}_{n}: A \longmapsto X_{n-1} \rightarrow H_{n-1}, \cdots, \mathrm{C}_{2}: H_{2} \longmapsto$ $X_{1} \rightarrow H_{1}$ and $\mathrm{C}_{1}: H_{1} \longmapsto X_{0} \rightarrow Z$

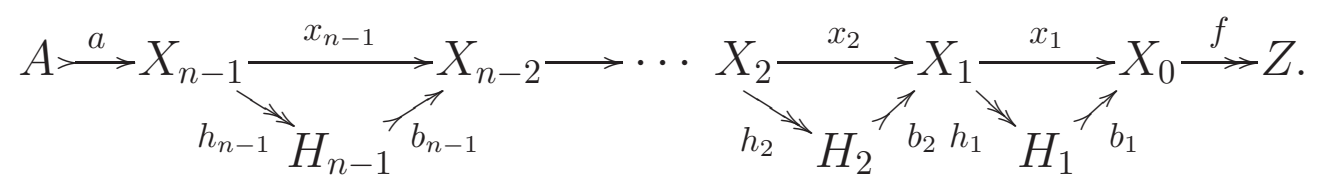

written $\mathrm{E}=\mathrm{C}_{n} \cdots \mathrm{C}_{1}$ and called the Yoneda composition of $\mathrm{C}_{n}, \cdots, \mathrm{C}_{1}$. In this case we have $\mathrm{E} \zeta=\mathrm{C}_{\mathrm{n}} \cdots \mathrm{C}_{2}\left(\mathrm{C}_{1} \zeta\right)$ and $\alpha \mathrm{E}=\left(\alpha \mathrm{C}_{\mathrm{n}}\right) \mathrm{C}_{n-1} \cdots \mathrm{C}_{1}$.

4.4. Semi-direct products. The notion of semi-direct products was introduced in [12] for Barr-exact and protomodular categories with certain colimits. The presence of all finite colimits simplifies its definition, so we will describe semi-direct products in any semi-abelian category $\mathcal{S}$.

We denote the injections of a coproduct by $i_{X}: X \rightarrow X+Y, \cdots$ or $i_{0}, i_{1}$ : $X \rightarrow X+X, \cdots$.

Theorem 4.12. ([12]) For every morphism $v: U \rightarrow V$ in $\mathcal{S}$, the change of base functor, with respect to the fibration of pointed objects $\Pi: \operatorname{Pt} \mathcal{S} \rightarrow \mathcal{S}$, $v^{*}: \operatorname{Pt} \mathcal{S}[V] \rightarrow \operatorname{Pt} \mathcal{S}[U]$ is monadic.

Given a fixed object $X_{0}$ of $\mathcal{S}$, we have an adjunction

$$
\begin{aligned}
& \left(0_{X_{0}}\right)^{!}: \mathcal{S} \longrightarrow \operatorname{Pt} \mathcal{S}\left[X_{0}\right] \quad \dashv \quad\left(0_{X_{0}}\right)^{*}: \operatorname{Pt} \mathcal{S}\left[X_{0}\right] \quad \longrightarrow \mathcal{S}, \\
& X_{1} \longmapsto X_{1}+X_{0} \underset{i_{X_{0}}}{\stackrel{<0,1>}{\rightleftarrows}} X_{0} \quad W \underset{t}{\stackrel{g}{\rightleftarrows}} X_{0} \longmapsto \mathrm{k}(W)
\end{aligned}
$$


and an induced monad in $\mathcal{S}$ represented by $\Pi^{X_{0}}$. A $\Pi^{X_{0}}$-algebra $\left(X_{1}, \xi_{X_{1}}\right.$ : $\left.\mathrm{k}\left(X_{1}+X_{0}\right) \rightarrow X_{1}\right)$ is also called an $X_{0}$-algebra. By Theorem 4.12 , the comparison functor $\operatorname{Pt} \mathcal{S}\left[X_{0}\right] \cong \mathcal{S}^{\Pi^{X_{0}}}$ is an equivalence.

Definition 4.13. The semi-direct product $\left(X_{1}, \xi_{X_{1}}\right) \rtimes X_{0}$ of an $X_{0}$-algebra $\left(X_{1}, \xi_{X_{1}}\right)$ and $X_{0}$ is the domain of the object in $\operatorname{Pt} \mathcal{S}\left[X_{0}\right]$ that corresponds to $\left(X_{1}, \xi_{X_{1}}\right)$ through the previous equivalence $\operatorname{Pt} \mathcal{S}\left[X_{0}\right] \cong \mathcal{S}^{\Pi^{X_{0}}}$.

Given an $X_{0}$-algebra $\left(X_{1}, \xi_{X_{1}}\right)$, there exists an object $\left(X_{1}, \xi_{X_{1}}\right) \rtimes X_{0} \rightleftarrows X_{0}$ $\in \operatorname{Pt} \mathcal{S}\left[X_{0}\right]$ such that the top and bottom squares are pullbacks and pushouts in

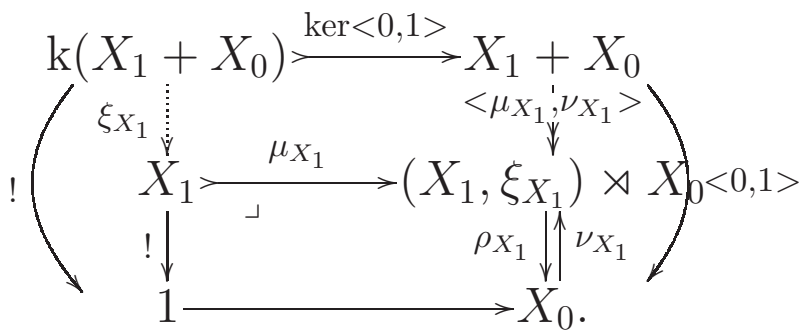

Moreover, for an object $W \underset{\nu}{\rightleftarrows} X_{0}$ in PtS $\left[X_{0}\right]$ with kernel $\mu: X_{1} \longmapsto W$, we have $W \cong\left(X_{1}, \xi_{X_{1}}\right) \rtimes X_{0}$ for the $X_{0}$-algebra $\left(X_{1}, \xi_{X_{1}}=\mathrm{k}<\mu, \nu>\right)$.

Notations 4.14. Let $\left(X_{1}, \xi_{X_{1}}\right)$ be an $X_{0}$-algebra and $\left(X_{1}^{\prime}, \xi_{X_{1}^{\prime}}\right)$ an $X_{0}^{\prime}$-algebra.

1: Given morphisms $\theta_{1}: X_{1} \rightarrow X_{1}^{\prime}$ and $\theta_{0}: X_{0} \rightarrow X_{0}^{\prime}$ such that $\theta_{1} \cdot \xi_{X_{1}}=$ $\xi_{X_{1}^{\prime}} \cdot \mathrm{k}\left(\theta_{1}+\theta_{0}\right)$, we have a commutative diagram

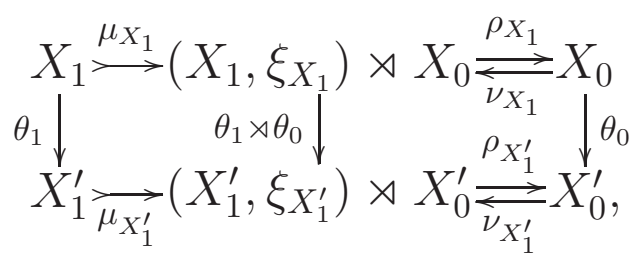

where $\theta_{1} \rtimes \theta_{0}$ is induced by the top pushout in (7).

2: We have a product $\left(X_{0} \times X_{0}^{\prime}\right)$-algebra $\left(X_{1} \times X_{1}^{\prime}, \xi_{X_{1} \times X_{1}^{\prime}}\right)$, for $\xi_{X_{1} \times X_{1}^{\prime}}=$ $<\xi_{X_{1}} \cdot \mathrm{k}\left(p_{X_{1}}+p_{X_{1}}\right), \xi_{X_{1}^{\prime}} \cdot \mathrm{k}\left(p_{X_{1}^{\prime}}+p_{X_{1}^{\prime}}\right)>$. When $X_{0}=X_{0}^{\prime}$, the product $X_{0^{-}}$-algebra is given by $\xi_{X_{1} \times X_{1}^{\prime}}=<\xi_{X_{1}} \cdot \mathrm{k}\left(p_{X_{1}}+1_{X_{0}}\right), \xi_{X_{1}^{\prime}} \cdot \mathrm{k}\left(p_{X_{1}^{\prime}}+1_{X_{0}}\right)>$. 3: A $Z$-module in $\mathcal{M}$ is represented by a diagram of the form

$$
\mathrm{O}_{A}: A \stackrel{\mu_{A}}{\longrightarrow}\left(A, \xi_{A}\right) \rtimes Z \underset{\nu_{A}}{\stackrel{\rho_{A}}{\rightleftarrows}} Z
$$

or just by the $Z$-algebra $\left(A, \xi_{A}\right)$. To simplify, we denote a morphism $\left(\alpha, \alpha \rtimes 1_{Z}, 1_{Z}\right): \mathrm{O}_{A} \rightarrow \mathrm{O}_{A^{\prime}}$ just by $\alpha: A \rightarrow A^{\prime}$. 


\section{Baer sum of singular extensions}

In this section we generalize the Baer Extension Theory for groups (or Lie algebras) of order 1 to the context of Moore categories. To do so, we apply the direction functor theory of order 1 (Subsection 3.1) to the Barr-exact category with finite limits $\mathcal{E}=\mathcal{M} / Z$.

5.1. The direction of singular extensions. The kernel pair of a morphism $f: X \rightarrow Z$ is denoted by $\left(f_{0}, f_{1}: X \times_{Z} X \rightarrow X\right)$.

Proposition 5.1. The direction functor $d: \operatorname{Sing}_{Z}(\mathcal{M}) \rightarrow \operatorname{Mod}_{Z}(\mathcal{M})$ assigns to each singular extension in $\mathcal{M}$ of $A$ by $Z$ a $Z$-module structure on $A$.

Proof: Using the equivalences (5) and (6), the direction functor is defined by

$$
\begin{aligned}
d: \operatorname{Sing}_{Z}(\mathcal{M}) & \longrightarrow \operatorname{Mod}_{Z}(\mathcal{M}) . \\
\mathrm{E}: A \stackrel{a}{\longrightarrow} X \stackrel{f}{\longrightarrow} Z & \longmapsto \mathrm{O}_{f}: A \stackrel{\mu_{f}}{\longrightarrow}\left(A, \xi_{f}\right) \rtimes Z \underset{\rho_{f}}{\stackrel{\rho_{f}}{\rightleftarrows}} Z
\end{aligned}
$$

The $Z$-module $\mathrm{O}_{f}$ is characterized by the following downward pullback

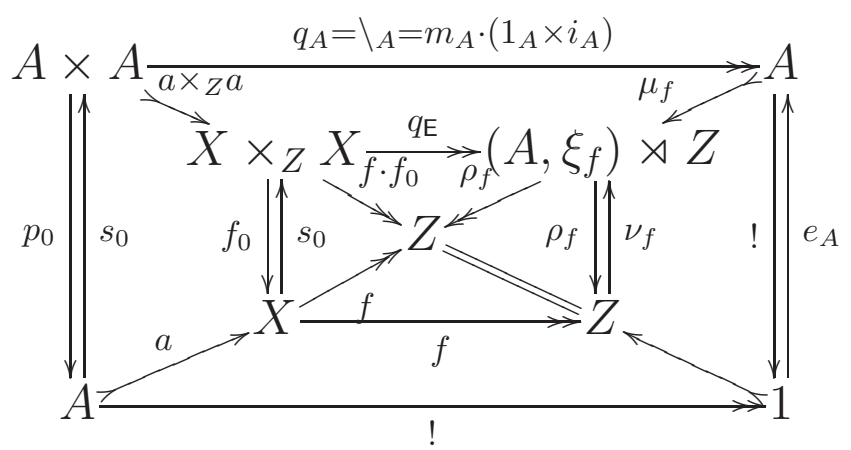

or, equivalently, by the upward pushout, both in $\operatorname{Sing}_{Z}(\mathcal{M})$; it corresponds to the diagram of Definition 3.4 for $\operatorname{AutM}\left((\mathcal{M} / Z)_{\#}\right)$.

5.2. The Baer sum of singular extensions. Let $\mathrm{O}_{A}$ be a fixed $Z$-module as in (8). The objects of the groupoid $d^{-1}\left(\mathrm{O}_{A}\right)$ are the singular extensions $\mathrm{E}: A \longmapsto X \stackrel{f}{\rightarrow} Z$ in $\mathcal{M}$ of $A$ by $Z$ such that $\mathrm{O}_{f} \cong \mathrm{O}_{A}$, i.e. $\left(A, \xi_{f}\right) \cong\left(A, \xi_{A}\right)$. We use the classical notation $\operatorname{Opext}\left(Z, A, \mathrm{O}_{A}\right)$ to represent the abelian group of the equivalence classes of singular extensions in $d^{-1}\left(\mathrm{O}_{A}\right)$. The first cohomology group of the $Z$-module $A$ is (see (4))

$$
\mathbb{H}_{\mathcal{M} / Z}^{1}(A) \cong \pi_{0}\left(d^{-1}\left(\mathrm{O}_{A}\right)\right)=\operatorname{Opext}\left(Z, A, \mathrm{O}_{A}\right)
$$


The group operation of $\mathbb{H}_{\mathcal{M} / Z}^{1}(A)$, induced by the tensor product of the monoidal structure of $d^{-1}\left(\mathrm{O}_{A}\right)$, is called the Baer sum, and is defined as $[E]_{\sim}+\left[E^{\prime}\right]_{\sim}=\left[E \otimes_{1} E^{\prime}\right]_{\sim}$. The singular extension $E \otimes_{1} E^{\prime}$ is the codomain of the $d$-cocartesian morphism $\bar{\mu}_{\mathrm{E}, \mathrm{E}^{\prime}}$ above the multiplication $m_{\mathrm{O}_{A}}$ with domain the product $E \times_{Z} E^{\prime}\left(=\left(E \times E^{\prime}\right) \Delta_{Z}\right)$ in $\operatorname{Sing}_{Z}(\mathcal{M})$ (Remark 3.5.3.)

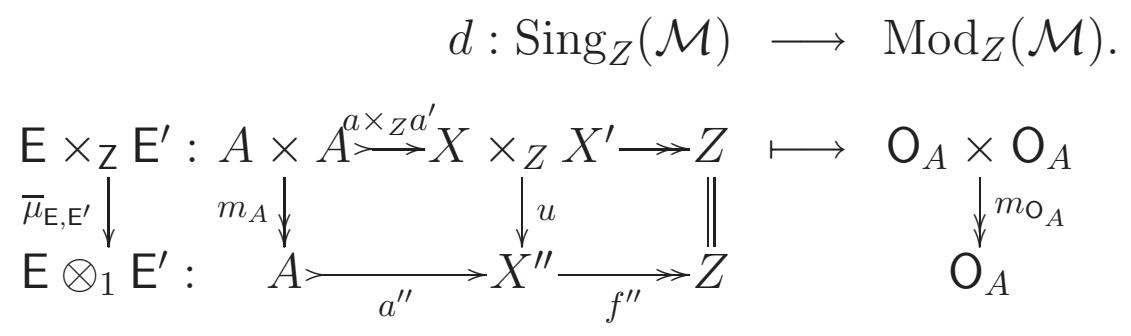

By Remark 4.9.2., $u$ is a regular epimorphism and, by Remark 4.9.1., the left square is a pullback, thus a pushout. So, the tensor product is given by $\mathrm{E} \otimes_{1} \mathrm{E}^{\prime}=m_{A}\left(\mathrm{E} \times \mathrm{E}^{\prime}\right) \Delta_{Z}$, just like the Baer sum of singular extensions of groups $([23])$.

5.3. The 6-term exact sequence. Given a short exact sequence

$$
\mathrm{S}: \mathrm{O}_{N} \stackrel{k}{\longrightarrow} \mathrm{O}_{P} \stackrel{p}{\longrightarrow} \mathrm{O}_{Q}
$$

in $\operatorname{Mod}_{Z}(\mathcal{M})$, the induced 6-term exact sequence is

$$
\begin{aligned}
0 \longrightarrow \operatorname{Hom}_{\mathcal{M} / Z}\left(1_{Z}, \rho_{N}\right) \stackrel{k_{*}}{\longrightarrow} \operatorname{Hom}_{\mathcal{M} / Z}\left(1_{Z}, \rho_{P}\right) \stackrel{p_{*}}{\longrightarrow} \operatorname{Hom}_{\mathcal{M} / Z}\left(1_{Z}, \rho_{Q}\right) \stackrel{\delta^{0}}{\longrightarrow} \\
\longrightarrow \operatorname{Opext}\left(Z, N, \mathrm{O}_{N}\right) \underset{k_{*}^{1}}{\longrightarrow} \operatorname{Opext}\left(Z, P, \mathrm{O}_{P}\right) \underset{p_{*}^{1}}{\longrightarrow} \operatorname{Opext}\left(Z, Q, \mathrm{O}_{Q}\right) .
\end{aligned}
$$

The connection morphism is defined by (Theorem 2.8)

$$
\begin{aligned}
\delta^{0}: \operatorname{Hom}_{\mathcal{M} / Z}\left(1_{Z}, \rho_{Q}\right) & \longrightarrow \operatorname{Opext}\left(Z, N, \mathrm{O}_{N}\right), \\
Z \underset{Z_{0}}{\longrightarrow}\left(Q, \xi_{Q}\right) \rtimes Z & \longmapsto\left[\mathrm{J} c_{0}: N \stackrel{b_{1}}{\longrightarrow} X \stackrel{f}{\longrightarrow} Z\right]_{\sim}
\end{aligned}
$$


where the singular extension $\delta^{0}\left(c_{0}\right)$ is obtained from the pullback

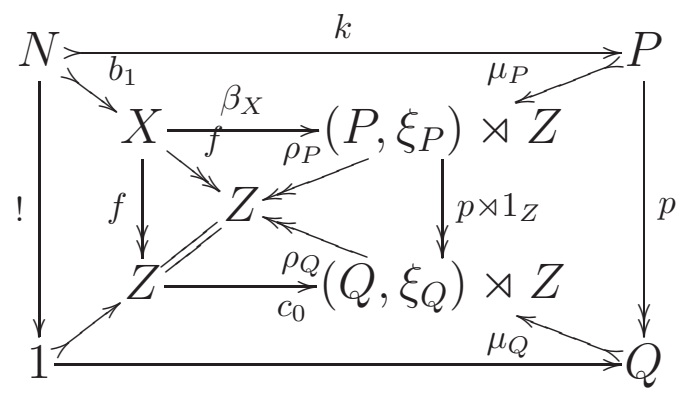

in $\operatorname{Sing}_{Z}(\mathcal{M})$. On the other hand, using this pullback it is easy to see that the singular extension $\delta^{0}\left(c_{0}\right)$ is also given by the composition $\mathrm{J} c_{0}$

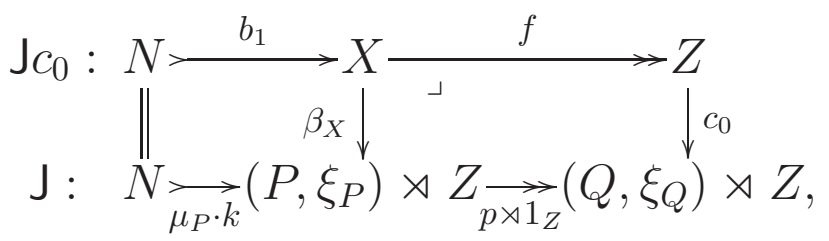

just like the definition of connecting morphism for groups ([23]).

\section{Baer sum of crossed 2-fold extensions}

In this section we generalize the Baer Extension Theory for groups (or Lie algebras) of order 2 to the context of Moore categories. But before, we must define the notions of an internal crossed module and that of a crossed 2-fold extension for these categories. As for groups, these notions are related to that of internal groupoids. Then we apply the direction functor theory of order 2 (Subsection 3.2) to the Barr-exact category with finite limits $\mathcal{E}=\mathcal{M} / Z$.

6.1. Internal crossed modules. The notion of a crossed module is extended to the context of any semi-abelian category $\mathcal{S}$. We recall the main considerations of [22].

Definition 6.1. An internal crossed module $\chi_{1}:\left(X_{1}, \xi_{X_{1}}\right) \rightarrow X_{0}$ in $\mathcal{S}$ is composed by an $X_{0}$-algebra $\left(X_{1}, \xi_{X_{1}}\right)$ and a morphism $\chi_{1}$ such that the following diagrams are commutative

$$
\begin{array}{cccc}
\mathrm{k}\left(X_{1}+X_{0}\right) \stackrel{\mathrm{ker}<0,1>}{\longrightarrow} X_{1}+X_{0} & \mathrm{k}\left(X_{1}+\left(X_{1}+X_{0}\right)\right) \stackrel{\mathrm{k}\left(1+<\chi_{1}, 1>\right)}{\longrightarrow} \mathrm{k}\left(X_{1}+X_{0}\right) \\
\xi_{X_{1}} \downarrow & \mathrm{A} & \downarrow<\chi_{1}, 1> & \mathrm{k}<i_{0}, 1>\downarrow \\
X_{1} \longrightarrow X_{0} & \mathrm{k}\left(X_{1}+X_{0}\right) \stackrel{\mathrm{B}}{\chi_{1}} \underset{\xi_{X_{1}}}{\longrightarrow} X_{1} .
\end{array}
$$


A morphism of internal crossed modules in $\mathcal{S}$ is given by morphisms $\beta$ and $\gamma$ making the following diagrams commute

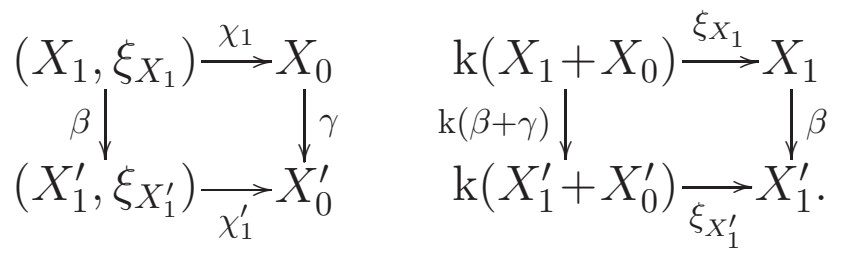

We represent by $\mathrm{XMod}(\mathcal{S})$ the category of internal crossed modules in $\mathcal{S}$.

The well known equivalence between crossed modules and internal groupoids for groups is extended to the context of semi-abelian categories.

Proposition 6.2. ([22]) The equivalence $\operatorname{XMod}(\mathcal{S}) \cong \operatorname{Grd}(\mathcal{S})$ holds.

Proof: We omit some of the technical details of the proof. The simplifications mentioned in Remark 4.3.2. will be used.

We define a functor

$$
\begin{aligned}
& F: \operatorname{XMod}(\mathcal{S}) \longrightarrow \operatorname{Grd}(\mathcal{S}), \\
& \left(X_{1}, \xi_{X_{1}}\right) \stackrel{\chi_{1}}{\longrightarrow} X_{0} \longmapsto\left(X_{1}, \xi_{X_{1}}\right) \rtimes X_{0} \underset{\frac{\rho_{X_{1}}}{\kappa_{X_{1}} \rightrightarrows}}{\sigma_{X_{1}}} X_{0} \\
& (\beta, \gamma) \longmapsto(\beta \rtimes \gamma, \gamma)
\end{aligned}
$$

where the commutativity of (10 A) and the top pushout in (7) give a unique morphism in

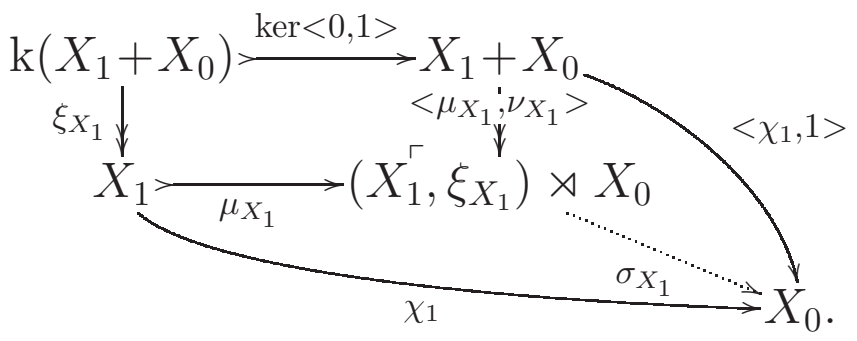

It is easy to see that $\left(X_{1}, \xi_{X_{1}}\right) \rtimes X_{0} \underset{0}{\frac{\rho_{X_{1}}}{\sigma_{X_{1}} \rightrightarrows}} X_{0}$ is a reflexive graph. The commutativity of (10 B) guarantees the existence of the composition morphism which makes the reflexive graph multiplicative, thus an internal groupoid. Since $(\beta \rtimes \gamma, \gamma)$ is a morphism of reflexive graphs it is also an internal functor between the internal groupoids they support. 
We also define a functor

$$
\begin{aligned}
G: \operatorname{Grd}(\mathcal{S}) & \longrightarrow \operatorname{XMod}(\mathcal{S}), \\
X_{1} \underset{c}{\stackrel{d}{\rightleftarrows}} X_{0} & \longmapsto\left(D=\operatorname{ker}(d), \xi_{D}=\mathrm{k}<\operatorname{ker}(d), e>\right) \stackrel{c \cdot \operatorname{ker}(d)}{\longrightarrow} X_{0} \\
\left(\varphi_{1}, \varphi_{0}\right) & \longmapsto\left(\mathrm{k}\left(\varphi_{1}\right), \varphi_{0}\right)
\end{aligned}
$$

where the equality $c \cdot \operatorname{ker}(d) \cdot \mathrm{k}<\operatorname{ker}(d), e>=c \cdot<\operatorname{ker}(d), e>\cdot \operatorname{ker}<$ $0,1>=<c \cdot \operatorname{ker}(d), 1>\cdot \operatorname{ker}<0,1>$ proves the commutativity of (10 A). Moreover, the composition morphism of the internal groupoid guarantees the commutativity of (10 B). The definition of $G$ on morphism is as expected and the proof that $G\left(\varphi_{1}, \varphi_{0}\right) \in \operatorname{XMod}(\mathcal{S})$ is straightforward.

Given an internal crossed module $\chi_{1}:\left(X_{1}, \xi_{X_{1}}\right) \rightarrow X_{0}$, it is easy to show that $G\left(F\left(\chi_{1}\right)\right) \cong \chi_{1}$ since $\chi_{1}=\sigma_{X_{1}} \cdot \mu_{X_{1}}$, by the definition of $\sigma_{X_{1}}$. For every internal groupoid $\underline{X}_{1} \in \operatorname{Grd}(\mathcal{S})$, we have $F\left(G\left(\underline{X}_{1}\right)\right) \cong \underline{X}_{1}$ since the split extension $D \stackrel{\operatorname{ker}(d)}{\longmapsto} X_{1} \stackrel{\stackrel{d}{\rightleftarrows}}{\rightleftarrows} X_{0}$ produces an isomorphism $\left(D, \xi_{D}\right) \rtimes X_{0} \cong X_{1}$ (see the observation after diagram (7)).

Proposition 6.3. Let $\chi_{1}:\left(X_{1}, \xi_{X_{1}}\right) \rightarrow X_{0}$ be an internal crossed module in $\mathcal{S}$. By taking $A=\operatorname{ker}\left(\chi_{1}\right)$ and $Z=\operatorname{coker}\left(\chi_{1}\right)$, we have an exact sequence

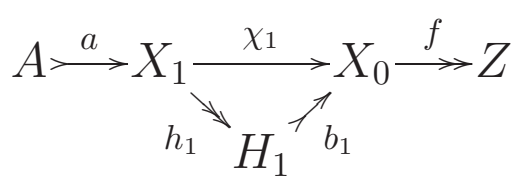

such that $A \in \mathrm{Ab}(\mathcal{S})$ and $h_{1}$ has a central kernel relation.

Proof: By Proposition 6.2, the internal crossed module is equivalent to an internal groupoid; we use the same notations. We begin by noticing that $Z$ is also the co-equalizer of $\left(\rho_{X_{1}}, \sigma_{X_{1}}\right)$. Using the fact that $<\mu_{X_{1}}, \nu_{X_{1}}>$ is an epimorphism and the equality $f \cdot \rho_{X_{1}} \cdot<\mu_{X_{1}}, \nu_{X_{1}}>=f \cdot<0,1>=f \cdot<$ $\chi_{1}, 1>=f \cdot \sigma_{X_{1}} \cdot\left\langle\mu_{X_{1}}, \nu_{X_{1}}>\right.$, we conclude that $f \cdot \rho_{X_{1}}=f \cdot \sigma_{X_{1}}$. Then, the whole commutative diagram

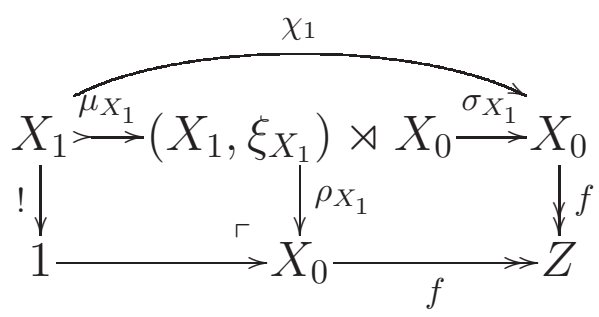

and the left square are pushouts, thus the right square is a pushout. 
Next, we see that $\chi_{1}$ admits a (regular epimorphism, kernel) factorization. For that we consider $H_{1}=\operatorname{ker}(f)$, the induced morphism $h_{1}$ and apply Remark 4.9.3. to the commutative diagram

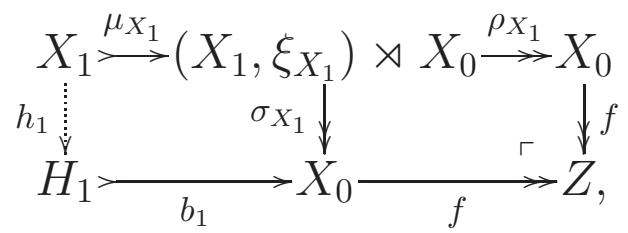

to conclude that $h_{1}$ is a regular epimorphism. So, by taking $A=\operatorname{ker}\left(\chi_{1}\right)$, we get the above mentioned exact sequence.

Let $\left(\pi_{1}, \pi_{2}\right)$ be the pullback of $\left(\rho_{X_{1}}, \sigma_{X_{1}}\right), m$ the composition of the internal groupoid and consider the unique morphism in

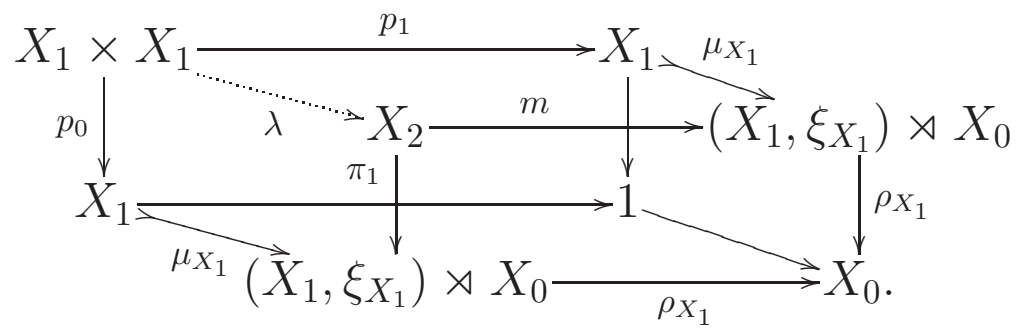

The front, right and back faces are pullbacks, implying that the left face is a pullback. In the commutative diagram

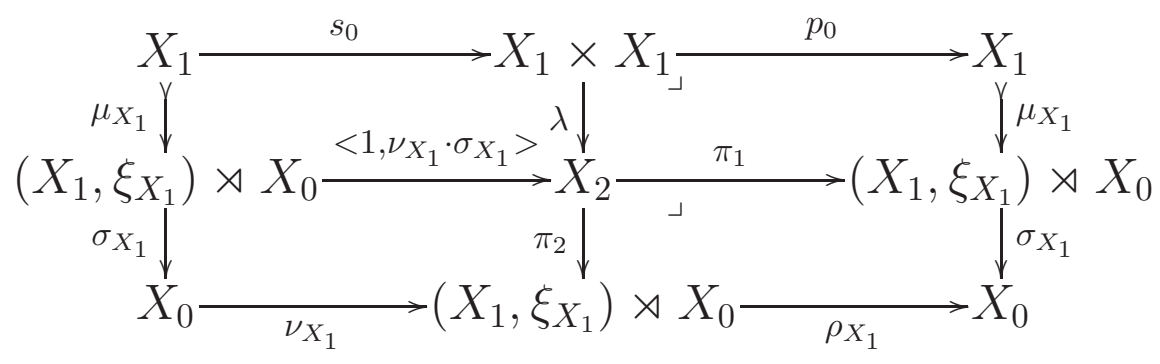

the right side and the whole diagram are pullbacks, thus the left side is a pullback. Finally, since $A=\operatorname{ker}\left(\chi_{1}=\sigma_{X_{1}} \cdot \mu_{X_{1}}\right)$, we conclude that $\langle a, a\rangle$ : $A \rightarrow X_{1} \times X_{1}$ is a kernel. Hence, $\left\langle 1_{A}, 1_{A}>: A \rightarrow A \times A\right.$ is a kernel, guaranteing that $A \in \operatorname{Ab}(\mathcal{S})$.

To finish, $h_{1}$ has a central kernel relation because the composition $A \stackrel{a}{\longmapsto}$ $X_{1} \stackrel{s_{0}}{\rightarrow} X_{1} \times X_{1}$ is a kernel (Theorem 5.2 of [13]).

Remark 6.4. To conclude that an internal crossed module $\chi_{1}:\left(X_{1}, \xi_{X_{1}}\right) \rightarrow$ $X_{0}$ induces a $Z$-module structure on its kernel $A$, for $Z=\operatorname{coker}\left(\chi_{1}\right)$, (as 
in the case of groups) we must appeal to the second order direction functor when considering Moore categories (Proposition 6.9).

\subsection{Crossed 2-fold extensions.}

Definition 6.5. A crossed 2 -fold extension in $\mathcal{S}$ of $A$ by $Z$ is an exact sequence $\mathrm{E}: A \longmapsto\left(X_{1}, \xi_{X_{1}}\right) \rightarrow X_{0} \rightarrow Z$ in $\mathcal{S}$ with $\left(X_{1}, \xi_{X_{1}}\right) \rightarrow X_{0}$ an internal crossed module in $\mathcal{S}$. A morphism of crossed 2-fold extensions in $\mathcal{S}$ is given by morphisms $\alpha, \beta, \gamma$ and $\zeta$ making the following diagram commutative

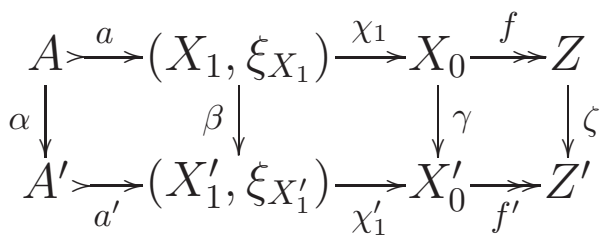

such that $(\beta, \gamma) \in \operatorname{XMod}(\mathcal{S})$.

We represent by $2-\mathrm{XMod}_{Z}(\mathcal{S})$ the category of crossed 2 -fold extensions in $\mathcal{S}$ over $Z$. The terminal object of $2-\operatorname{XMod}_{Z}(\mathcal{S})$ is $Z: 1=1 \longmapsto Z=Z$. We denote the product of $\mathrm{E}, \mathrm{E}^{\prime} \in 2-\operatorname{XMod}_{Z}(\mathcal{S})$ by $\mathrm{E} \times_{\mathrm{Z}} \mathrm{E}^{\prime}: A \times A^{\prime} \longmapsto$ $\left(X_{1} \times X_{1}^{\prime}, \xi_{X_{1} \times X_{1}^{\prime}}\right) \rightarrow X_{0} \times_{Z} X_{0}^{\prime} \rightarrow Z$, where $\xi_{X_{1} \times X_{1}^{\prime}}=<\xi_{X_{1}} \cdot \mathrm{k}\left(p_{X_{1}}+p_{X_{0}}\right), \xi_{X_{1}^{\prime}}$. $\mathrm{k}\left(p_{X_{1}^{\prime}}+p_{X_{0}^{\prime}}\right)>$.

The equivalence $\operatorname{XMod}(\mathcal{S}) \cong \operatorname{Grd}(\mathcal{S})$ allows us to establish the following:

Proposition 6.6. The equivalence $2-\operatorname{XMod}_{Z}(\mathcal{S}) \cong \operatorname{Asp}(\mathcal{S} / Z)$ holds.

Proof: The simplifications mentioned in Remark 4.3.2. will be used.

An aspherical internal groupoid in $\mathcal{S} / Z$ consists of a commutative diagram of the form

$$
\underline{X}_{1, f}: \quad \underset{f \cdot d=f \cdot c}{\stackrel{c}{c} \underset{Z^{2} \longleftarrow_{f}}{\rightleftarrows}} X_{0},
$$

equipped with a composition morphism $m$, where the top line $\underline{X}_{1}: X_{1} \stackrel{\rightrightarrows}{\rightarrow} X_{0}$, with $m$, is an internal groupoid in $\mathcal{S}$ and $f: X_{0} \rightarrow Z$ and $\langle d, c\rangle: X_{1} \rightarrow$ $X_{0} \times_{Z} X_{0}$ are regular epimorphisms. 
The functors that compose this equivalence are based on those of Proposition 6.2 and we use the same notations as in Proposition 6.3. We define

$$
F_{2}: 2-\operatorname{XMod}_{Z}(\mathcal{S}) \longrightarrow \operatorname{Asp}(\mathcal{S} / Z) .
$$

$$
\begin{aligned}
\mathrm{E}: A \stackrel{a}{\longrightarrow}\left(X_{1}, \xi_{X_{1}}\right) \stackrel{\chi_{1}}{\longrightarrow} X_{0} \stackrel{f}{\longrightarrow} Z & \longmapsto \\
\left(\alpha, \beta, \gamma, 1_{Z}\right) & \longmapsto(\beta \rtimes \gamma, \gamma) \\
1, \mathrm{E} & :\left(X_{1}, \xi_{X_{1}}\right) \underset{f \cdot \rho_{X_{1}}}{\rtimes X_{0} \frac{\rho_{X_{1}}}{{ }^{\nu} X_{1} \rightrightarrows}} X_{0} \\
\sigma_{X_{1}} \longleftarrow &
\end{aligned}
$$

We already know that the top line of $\underline{X}_{1, \mathrm{E}}$ is an internal groupoid by Proposition 6.2 and $f \cdot \rho_{X_{1}}=f \cdot \sigma_{X_{1}}$ (proof of Proposition 6.3). By applying Remark 4.9.2. to the commutative diagram

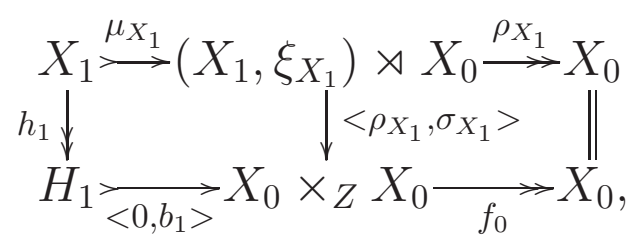

we conclude that $<\rho_{X_{1}}, \sigma_{X_{1}}>$ is a regular epimorphism.

We also define

$$
\begin{aligned}
G_{2}: \operatorname{Asp}(\mathcal{S} / Z) & \longrightarrow 2-X_{M} \operatorname{Mod}_{Z}(\mathcal{S}), \\
\underline{X}_{1, f}: X_{1} \stackrel{d}{\stackrel{c}{\rightleftarrows} X_{0}} & \longmapsto \mathrm{E}_{\underline{X}_{1, f}}: A \stackrel{a}{\longrightarrow}\left(D, \xi_{D}\right) \stackrel{c \cdot \operatorname{ker}(d)}{\longrightarrow} X_{0} \stackrel{f}{\longrightarrow} Z \\
Z^{\longleftarrow} & \\
\left(\varphi_{1}, \varphi_{0}, 1_{Z}\right) & \longmapsto\left(\mathrm{k}\left(\mathrm{k}\left(\varphi_{1}\right)\right), \mathrm{k}\left(\varphi_{1}\right), \varphi_{0}, 1_{Z}\right)
\end{aligned}
$$

for $D=\operatorname{ker}(d)$ and $A=\operatorname{ker}(c \cdot \operatorname{ker}(d))$. We already know that $c \cdot \operatorname{ker}(d)$ : $\left(D, \xi_{D}\right) \rightarrow X_{0}$ is an internal crossed module by Proposition 6.2. With the following pushouts, we see that $f=\operatorname{coker}(c \cdot \operatorname{ker}(d))$

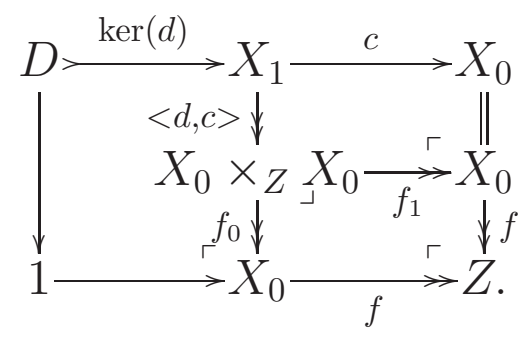

By Proposition 6.3, $\mathrm{E}_{\underline{X}_{1, f}}$ is an exact sequence.

Note that the definitions of $F_{2}$ and $G_{2}$ on morphisms are similar to those of $F$ and $G$, respectively. Moreover, it is easy to deduce that $G_{2} \cdot F_{2} \cong 1$ and $F_{2} \cdot G_{2} \cong 1$ from $G \cdot F \cong 1$ and $F \cdot G \cong 1$, respectively. 
Examples 6.7. The previous equivalence gives us:

$$
\begin{aligned}
& \text { 1: } \nabla_{1}(f): X_{0} \times_{Z} \underbrace{X_{0} \underset{Z}{\stackrel{f_{1}}{\leftrightarrows}} X_{f}}_{f \cdot f_{0}} X_{0} \sim \mathrm{E}_{\nabla_{1}(f)}: 1 \rightarrow\left(H_{1}, \xi_{H_{1}}\right) \stackrel{b_{1}}{\rightarrow} X_{0} \stackrel{f}{\rightarrow} Z \text {, for } \\
& H_{1}=\operatorname{ker}(f) \text { and } \xi_{H_{1}}=\mathrm{k}<<0, b_{1}>, s_{0}>\text {. }
\end{aligned}
$$

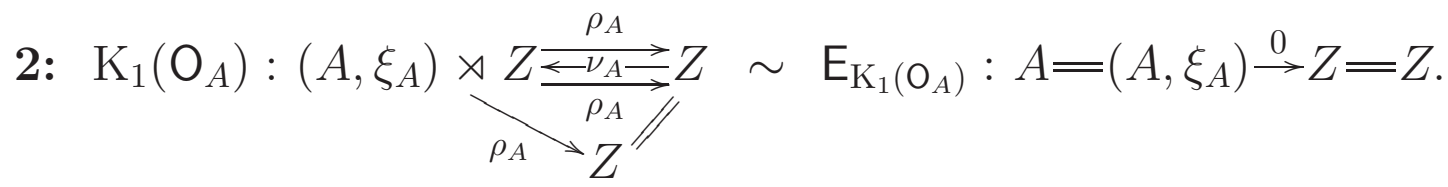

Remark 6.8. Consider $\underline{X}_{1, f} \sim \mathrm{E}_{\underline{X}_{1, f}}: A \stackrel{a}{\longmapsto}\left(X_{1}, \xi_{X_{1}}\right) \stackrel{\chi_{1}}{\rightarrow} X_{0} \stackrel{f}{\rightarrow} Z \in 2$ $\operatorname{XMod}_{Z}(\mathcal{S})$ decomposed as in Proposition 6.3. Since $h_{1}$ has a central kernel relation, then the kernel pair of $h_{1}$ has the form $\left(p_{X_{1}}, m_{h_{1}}: X_{1} \times A \rightarrow\right.$ $\left.X_{1}\right)$. So, the kernel pair $\underline{X}_{1, f} \times \nabla_{\nabla_{1}(f)} \underline{X}_{1, f} \sim \mathrm{E}_{\underline{X}_{1, f}} \times_{\mathrm{E}_{\nabla_{1}(f)}} \mathrm{E}_{\underline{X}_{1, f}}$ of the map $\left(!_{A}, h_{1}, 1_{X_{0}}, 1_{Z}\right): \mathrm{E}_{\underline{X}_{1, f}} \rightarrow \mathrm{E}_{\nabla_{1}(f)}$ is the crossed 2-fold extension given by $A \times A \stackrel{a \times 1_{A}}{\longrightarrow}\left(X_{1} \times A, \xi_{X_{1} \times A} \stackrel{\chi_{1} \cdot p_{X_{1}}}{\longrightarrow} X_{0} \stackrel{f}{\longrightarrow} Z\right.$, where $\xi_{X_{1} \times A}=<\xi_{X_{1}} \cdot \mathrm{k}\left(p_{X_{1}}+1_{X_{0}}\right), \xi_{X_{1}}$. $\mathrm{k}\left(m_{h_{1}}+1_{X_{0}}\right)>$.

6.3. The direction of crossed 2-fold extensions. With the notion of crossed 2-fold extensions and the equivalence $2-\operatorname{XMod}_{Z}(\mathcal{M}) \cong \operatorname{Asp}(\mathcal{M} / Z)$ we establish the link with the direction functor theory of order 2 .

Proposition 6.9. The second order direction functor $d_{2}: 2-\operatorname{XMod}_{Z}(\mathcal{M}) \rightarrow$ $\operatorname{Mod}_{Z}(\mathcal{M})$ assigns to each crossed 2-fold extension in $\mathcal{M}$ of $A$ by $Z$ a $Z$ module structure on $A$.

Proof: The category $\mathcal{M} / Z$ is protomodular, so $\operatorname{Grd}_{f}(\mathcal{M} / Z)$ is naturally Maltsev, for every object $f$ of $\mathcal{M} / Z$. Thus, all internal groupoids are necessarily abelian and by Proposition 6.6 we get the equivalence $2-\operatorname{XMod}_{Z}(\mathcal{M}) \cong$ $\operatorname{Asp}_{a b}(\mathcal{M} / Z)$. Using the equivalence of (6), the second order direction functor is defined by

$$
\begin{aligned}
& d_{2}: 2-\operatorname{XMod}_{Z}(\mathcal{M}) \longrightarrow \operatorname{Mod}_{Z}(\mathcal{M}) . \\
& \mathrm{E}_{\underline{X}_{1, f}}: A \stackrel{a}{\longrightarrow}\left(X_{1}, \xi_{X_{1}}\right) \stackrel{\chi_{1}}{\longrightarrow} X_{0} \stackrel{f}{\longrightarrow} Z \longmapsto\left(A, \xi_{\chi_{1}}\right)
\end{aligned}
$$


The $Z$-module $\left(A, \xi_{\chi_{1}}\right)$ is determined by the downward pullback

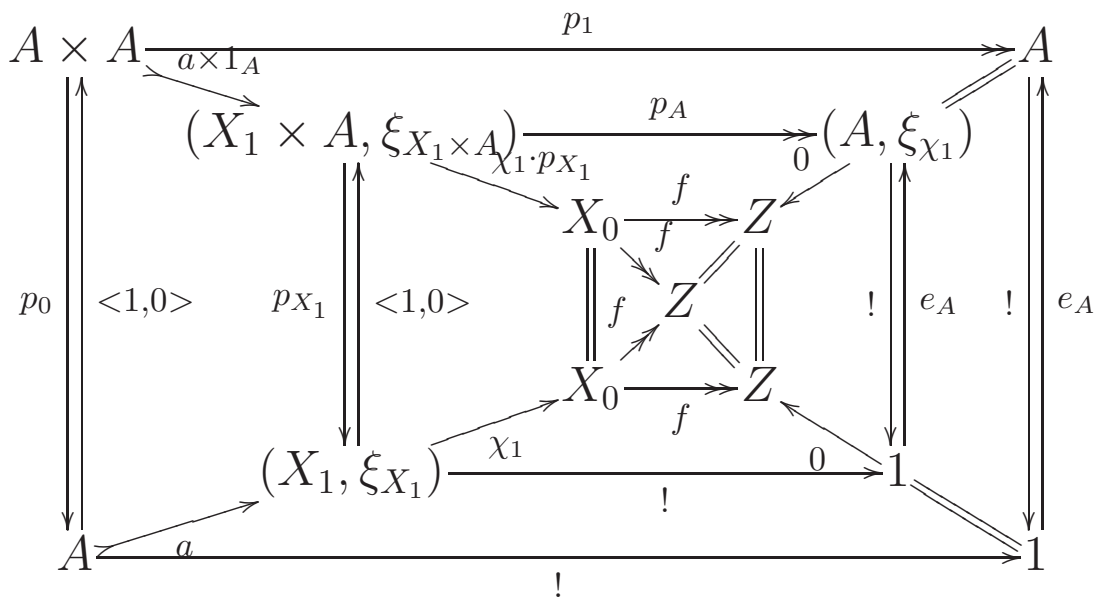

or, equivalently, by the upward pushout, both in $2-\operatorname{XMod}_{Z}(\mathcal{M})$; it corresponds to the diagram of Definition 3.7 for $\operatorname{Asp}_{a b}(\mathcal{M} / Z)$.

Remark 6.10. The top horizontal morphism of internal crossed modules in $\mathcal{M}$ given in (11) allows us to conclude that $\xi_{X_{1} \times A}$ defined in Remark 6.8 is also given by $<\xi_{X_{1}} \cdot \mathrm{k}\left(p_{X_{1}}+1_{X_{0}}\right), \xi_{A}^{X_{0}} \cdot \mathrm{k}\left(p_{A}+1_{X_{0}}\right)>$, for $\xi_{A}^{X_{0}}=\xi_{\chi_{1}} \cdot \mathrm{k}\left(1_{A}+f\right)$, i.e. $\xi_{X_{1} \times A}$ is actually a product $X_{0}$-algebra.

The Baer sum of crossed 2-fold extensions and the definition of the connecting morphism $\delta^{1}$ between levels 1 and 2 are included in the general treatment conducted in Section 7.

\section{Baer sum of crossed $n$-fold extensions}

In this section we generalize the Baer Extension Theory for groups (or Lie algebras) of order $n, n \geq 3$, to the context of Moore categories. For that we must define a crossed $n$-fold extension for these categories. As for groups, this notion is related to that of internal groupoids of higher dimensions. Then we apply the direction functor theory of order $n$ (Subsection 3.3) to the Barr-exact category with finite limits $\mathcal{E}=\mathcal{M} / Z$.

\subsection{Crossed $n$-fold extensions.}

Definition 7.1. A crossed $n$-fold extension in $\mathcal{S}$ of $A$ by $Z$ is an exact sequence $\mathrm{E}: A \stackrel{a}{\longmapsto} X_{n-1} \stackrel{\chi_{n-1}}{\longrightarrow} \cdots X_{2} \stackrel{\chi_{2}}{\rightarrow}\left(X_{1}, \xi_{X_{1}}\right) \stackrel{\chi_{1}}{\rightarrow} X_{0} \stackrel{f}{\rightarrow} Z$ in $\mathcal{S}$ with $\left(X_{1}, \xi_{X_{1}}\right) \rightarrow X_{0}$ an internal crossed module in $\mathcal{S}$ and $\left(X_{i}, \xi_{X_{i}}\right), \chi_{i} \in \operatorname{Mod}_{Z}(\mathcal{S})$, 
$2 \leq i \leq n-1$. A morphism of crossed $n$-fold extensions in $\mathcal{S}$ is given by morphisms $\alpha, \theta_{n-1}, \cdots, \theta_{2}, \beta, \gamma, \zeta$ making the following diagram commutative

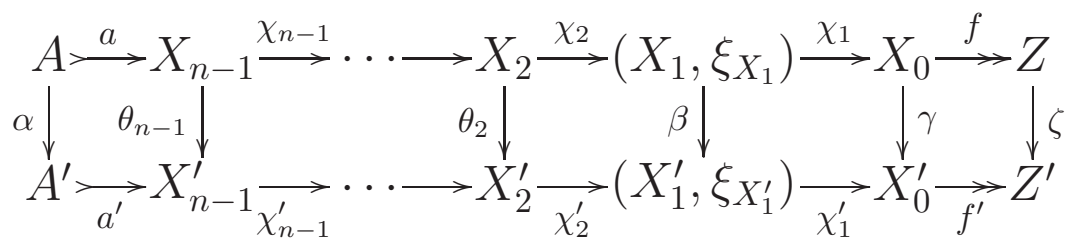

such that $(\beta, \gamma) \in \operatorname{XMod}(\mathcal{S})$.

We represent by $n-\mathrm{XMod}_{Z}(\mathcal{S})$ the category of crossed $n$-fold extensions in $\mathcal{S}$ over $Z$. The terminal object of $n-\operatorname{XMod}_{Z}(\mathcal{S})$ is $Z: 1=\cdots 1 \rightarrow Z=Z$. We denote the product of $\mathrm{E}, \mathrm{E}^{\prime} \in n-\operatorname{XMod}_{Z}(\mathcal{S})$ by $\mathrm{E} \times_{Z} \mathrm{E}^{\prime}: A \times A^{\prime} \longmapsto$ $X_{n-1} \times X_{n-1}^{\prime} \rightarrow \cdots\left(X_{1} \times X_{1}^{\prime}, \xi_{X_{1} \times X_{1}^{\prime}}\right) \rightarrow X_{0} \times_{Z} X_{0}^{\prime} \rightarrow Z$, where $\xi_{X_{1} \times X_{1}^{\prime}}=<$ $\xi_{X_{1}} \cdot \mathrm{k}\left(p_{X_{1}}+p_{X_{0}}\right), \xi_{X_{1}^{\prime}} \cdot \mathrm{k}\left(p_{X_{1}^{\prime}}+p_{X_{0}^{\prime}}\right)>$.

Remark 7.2. Every crossed $n$-fold extensions $\mathrm{E}$ in $\mathcal{S}$ can be decomposed into short exact sequences

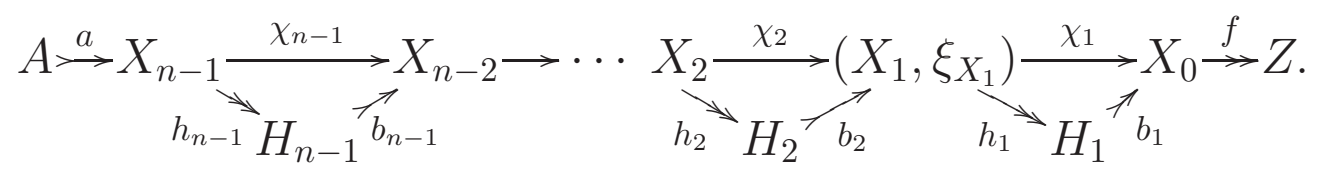

The second order direction applied to the crossed 2-fold extension at the end of (12) assigns to $H_{2}$ a $Z$-module structure $\left(H_{2}, \xi_{\chi_{1}}\right)$ in $\mathcal{S}$. The condition $\chi_{2} \in \operatorname{Mod}_{Z}(\mathcal{S})$ actually means that $h_{2} \in \operatorname{Mod}_{Z}(\mathcal{S})$. It is obvious that $H_{3}, \cdots$, $H_{n-1}, A$ and the morphisms between them belong to $\operatorname{Mod}_{Z}(\mathcal{S})$. Moreover, $\left(H_{2}, \xi_{H_{2}}\right)$ is an $X_{0}$-algebra for $\xi_{H_{2}}=\xi_{\chi_{1}} \cdot \mathrm{k}\left(1_{H_{2}}+f\right)$ and, for $\xi_{X_{2}}^{X_{0}}=\xi_{X_{2}} \cdot \mathrm{k}\left(1_{X_{2}}+\right.$ $f)$, we can deduce that $h_{2}$ is a morphism of $X_{0}$-algebras. The same holds for $H_{3}, X_{3}, \cdots, H_{n-1}, X_{n-1}, A$ and the morphisms between them.

We can extend the equivalence $2-\operatorname{XMod}_{Z}(\mathcal{S}) \cong \operatorname{Asp}(\mathcal{S} / Z)$ to higher orders:

Proposition 7.3. The equivalence $n-\operatorname{XMod}_{Z}(\mathcal{S}) \cong(n-1)-\operatorname{Asp}(\mathcal{S} / Z)$ holds for $n \geq 3$.

Proof: The simplifications mentioned in Remark 4.3.2. will be used. The proof of this proposition is made by induction on $n$.

We start with $n=3$. Using the equivalence $2-\operatorname{XMod}_{Z}(\mathcal{S}) \cong \operatorname{Asp}(\mathcal{S} / Z)$, an aspherical internal 2-groupoid consists of a commutative diagram of the 
form

$$
\underline{X}_{2, f}:\left(X_{2}, \xi_{X_{2}}\right) \underset{c}{\stackrel{d}{\rightleftarrows}}\left(X_{1}, \xi_{X_{1}}\right)
$$

such that the vertical diagrams on the right $\mathrm{k}\left(X_{1}\right) \stackrel{\operatorname{ker}\left(\chi_{1}\right)}{\longmapsto}\left(X_{1}, \xi_{X_{1}}\right) \stackrel{\chi_{1}}{\rightarrow} X_{0} \stackrel{f}{\rightarrow}$

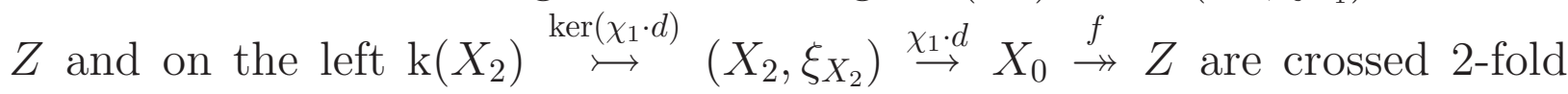
extensions, the top line $\left(\chi_{1} \cdot d\right) \underset{\rightarrow}{\leftrightarrows} \chi_{1}$, equipped with a composition morphism $m$, is an internal groupoid in $\operatorname{XMod}(\mathcal{S})$ and $\langle d, c\rangle: X_{2} \rightarrow X_{1} \times \mathrm{k}\left(X_{1}\right)$ is a regular epimorphism (where $\left(p_{X_{1}}, m_{h_{1}}: X_{1} \times \mathrm{k}\left(X_{1}\right) \rightarrow X_{1}\right)$ is the kernel pair of $\chi_{1}$; see Remark 6.8).

We define

$$
\begin{aligned}
& F_{3}: 3-\operatorname{XMod}_{Z}(\mathcal{S}) \longrightarrow 2-\operatorname{Asp}(\mathcal{S} / Z) .
\end{aligned}
$$

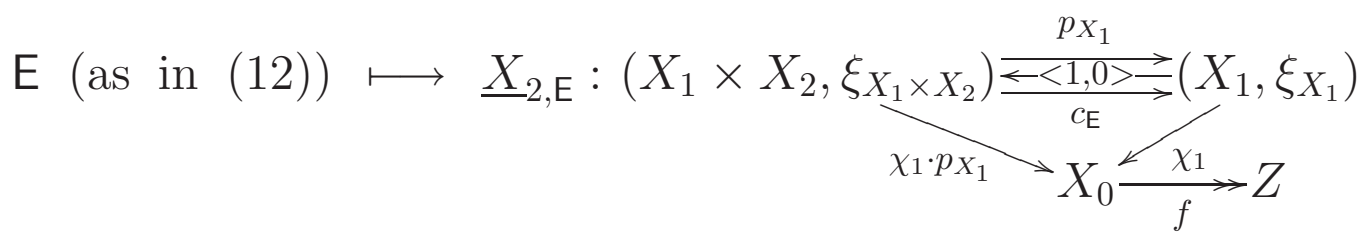

$$
\begin{aligned}
& \left(\alpha, \theta_{2}, \beta, \gamma, 1_{Z}\right) \longmapsto\left(\beta \times \theta_{2}, \beta, \gamma, 1_{Z}\right)
\end{aligned}
$$

Note that:

- both $H_{2} \stackrel{\operatorname{ker}\left(\chi_{1}\right)}{\longmapsto}\left(X_{1}, \xi_{X_{1}}\right) \stackrel{\chi_{1}}{\longrightarrow} X_{0} \stackrel{f}{\rightarrow} Z$ and $H_{2} \times X_{2} \stackrel{\operatorname{ker}\left(\chi_{1} \cdot p_{X_{1}}\right)}{\longmapsto}\left(X_{1} \times\right.$ $\left.X_{2}, \xi_{X_{1} \times X_{2}}\right) \stackrel{\chi_{1} \cdot p_{X_{1}}}{\rightarrow} X_{0} \stackrel{f}{\rightarrow} Z$ are in $2-\operatorname{XMod}_{Z}(\mathcal{S})$.

- $c_{\mathrm{E}}=m_{h_{1}} \cdot\left(1_{X_{1}} \times h_{2}\right) \in \mathrm{XMod}(\mathcal{S})$ since it is composed by $X_{0}$-algebras $m_{h_{1}}$ and $h_{2}$ (Remarks 6.8 and 7.2 , respectively).

$\cdot\left(\chi_{1} \cdot p_{X_{1}}\right) \underset{c_{\mathrm{E}}}{\stackrel{p_{X_{1}}}{\rightleftarrows<1,0>}} \chi_{1} \in \operatorname{Grd}\left(\operatorname{XMod}(\mathcal{S})\right.$ (with composition $\left.1 \times m_{X_{2}}\right)$.

- $<p_{X_{1}}, c_{\mathrm{E}}>=1 \times h_{2}: X_{1} \times X_{2} \rightarrow X_{1} \times H_{2}$ is a regular epimorphism.

- $\left(\beta \times \theta_{2}, \beta\right)$ is a morphism of reflexive graphs and $(\beta, \gamma),\left(\beta \times \theta_{2}, \gamma\right) \in$ $\operatorname{XMod}(\mathcal{S})$, thus $\left(\beta \times \theta_{2}, \beta, \gamma, 1_{Z}\right) \in 2-\operatorname{Asp}(\mathcal{S} / Z)$.

We also define

$$
\begin{aligned}
G_{3}: 2-\operatorname{Asp}(\mathcal{S} / Z) & \longrightarrow 3-\mathrm{XMod}_{Z}(\mathcal{S}), \\
\underline{X}_{2, f}(\operatorname{as~in~}(13)) & \longmapsto \mathrm{E}_{\underline{X}_{2, f}}: A \stackrel{a}{\longrightarrow} D \stackrel{\text { c.ker(d) }}{\longrightarrow}\left(X_{1}, \xi_{X_{1}}\right) \stackrel{\chi_{1}}{\longrightarrow} X_{0} \stackrel{f}{\longrightarrow} Z \\
\left(\varphi_{2}, \varphi_{1}, \gamma, 1_{Z}\right) & \longmapsto\left(\mathrm{k}\left(\mathrm{k}\left(\varphi_{2}\right)\right), \mathrm{k}\left(\varphi_{2}\right), \varphi_{1}, \gamma, 1_{Z}\right)
\end{aligned}
$$


for $D=\operatorname{ker}(d)$ and $A=\operatorname{ker}(c \cdot \operatorname{ker}(d))$. We already know that $\chi_{1} \in \operatorname{XMod}(\mathcal{S})$. To prove the exactness of $\mathrm{E}_{\underline{X}_{2, f}}$ it remains to show that the induced morphism $h: D \rightarrow \mathrm{K}\left(X_{1}\right)$ is a regular epimorphism. Since $\left\langle d, c>: X_{2} \rightarrow X_{1} \times \mathrm{k}\left(X_{1}\right)\right.$ is a regular epimorphism, we can use the following pushouts to conclude that $h_{1}$ is the co-equalizer of $(d, c)$

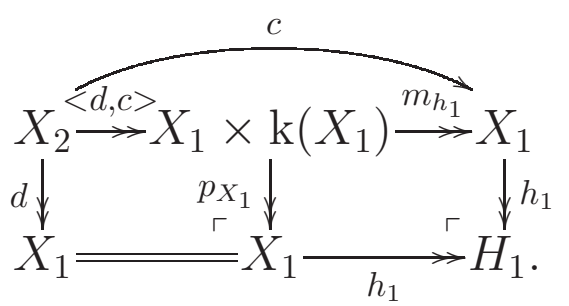

Then, by Remark 4.9.3. applied to the commutative diagram

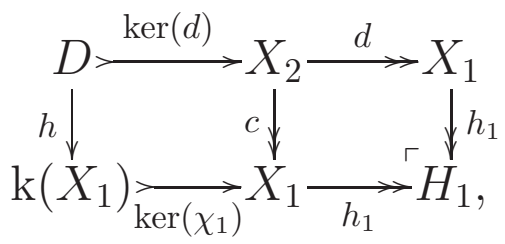

we conclude that $h$ is a regular epimorphism. Moreover, $D$ is a $Z$-module in $\mathcal{S}$ given by the following pullback in $\operatorname{Mod}_{Z}(\mathcal{S})$

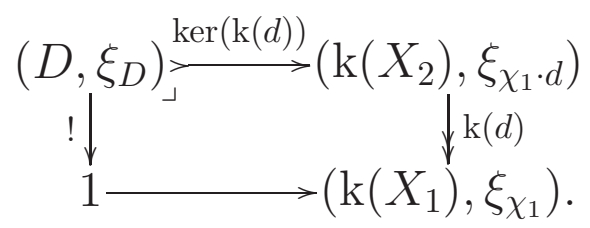

We can show that the previous diagram is a pullback, i.e. that $D$ is the kernel of $\mathrm{k}(d)$, by considering the commutative diagram

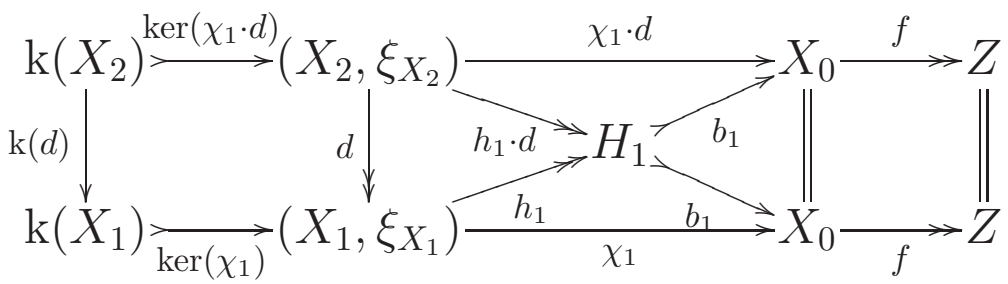

and applying Remark 4.9.1. to conclude that the left square is a pullback. Hence $\operatorname{ker}(\mathrm{k}(d)): D \longmapsto \mathrm{k}\left(X_{2}\right)$. Finally, $h:\left(D, \xi_{D}\right) \rightarrow\left(\mathrm{k}\left(X_{1}\right), \xi_{X_{1}}\right) \in$ $\operatorname{Mod}_{Z}(\mathcal{S})$ because $h=\mathrm{k}(c) \cdot \operatorname{ker}(\mathrm{k}(d))$, i.e. $h$ is composed by morphisms in $\operatorname{Mod}_{Z}(\mathrm{~S})$.

As for the definition of $G_{3}$ on morphisms, let $\mathrm{k}_{1}\left(\varphi_{2}\right):\left(\mathrm{k}\left(X_{2}\right), \xi_{\chi_{1} \cdot d}\right) \rightarrow$ $\left(\mathrm{k}\left(X_{2}^{\prime}\right), \xi_{\chi_{1}^{\prime} \cdot d^{\prime}}\right)$ be the unique map such that $\operatorname{ker}\left(\chi_{1}^{\prime} \cdot d^{\prime}\right) \cdot \mathrm{k}_{1}\left(\varphi_{2}\right)=\varphi_{2} \cdot \operatorname{ker}\left(\chi_{1} \cdot d\right)$ 
(using the left pullback in (14)). With the equality $\operatorname{ker}\left(\chi_{1} \cdot d\right) \cdot \operatorname{ker}(\mathrm{k}(d))=$ $\operatorname{ker}(d)$, we can prove that $\mathrm{k}_{1}\left(\varphi_{2}\right) \cdot \operatorname{ker}(\mathrm{k}(d))=\operatorname{ker}\left(\mathrm{k}\left(d^{\prime}\right)\right) \cdot \mathrm{k}\left(\varphi_{2}\right)$ and also

$$
\begin{aligned}
\operatorname{ker}\left(d^{\prime}\right) \cdot \mathrm{k}\left(\varphi_{2}\right) \cdot \xi_{D} & =\varphi_{2} \cdot \operatorname{ker}(d) \cdot \xi_{D} \\
& =\varphi_{2} \cdot \operatorname{ker}\left(\chi_{1} \cdot d\right) \cdot \operatorname{ker}(\mathrm{k}(d)) \cdot \xi_{D} \\
& =\operatorname{ker}\left(\chi_{1}^{\prime} \cdot d^{\prime}\right) \cdot \mathrm{k}_{1}\left(\varphi_{2}\right) \cdot \xi_{\chi_{1}} \cdot d \cdot \mathrm{k}\left(\operatorname{ker}(\mathrm{k}(d))+1_{Z}\right) \\
& =\operatorname{ker}\left(\chi_{1}^{\prime} \cdot d^{\prime}\right) \cdot \xi_{\chi_{1}^{\prime} \cdot d^{\prime}} \cdot \mathrm{k}\left(\left(\mathrm{k}_{1}\left(\varphi_{2}\right) \cdot \operatorname{ker}(\mathrm{k}(d))\right)+1_{Z}\right) \\
& =\operatorname{ker}\left(\chi_{1}^{\prime} \cdot d^{\prime}\right) \cdot \xi_{\chi_{1}^{\prime}} \cdot d^{\prime} \cdot \mathrm{k}\left(\left(\operatorname{ker}\left(\mathrm{k}\left(d^{\prime}\right)\right) \cdot \mathrm{k}\left(\varphi_{2}\right)\right)+1_{Z}\right) \\
& =\operatorname{ker}\left(\chi_{1}^{\prime} \cdot d^{\prime}\right) \cdot \operatorname{ker}\left(\mathrm{k}\left(d^{\prime}\right)\right) \cdot \xi_{D^{\prime}} \cdot \mathrm{k}\left(\mathrm{k}\left(\varphi_{2}\right)+1_{Z}\right) \\
& =\operatorname{ker}\left(d^{\prime}\right) \cdot \xi_{D^{\prime}} \cdot \mathrm{k}\left(\mathrm{k}\left(\varphi_{2}\right)+1_{Z}\right) .
\end{aligned}
$$

So, we conclude that $\mathrm{k}\left(\varphi_{2}\right) \cdot \xi_{D}=\xi_{D^{\prime}} \cdot \mathrm{k}\left(\mathrm{k}\left(\varphi_{2}\right)+1_{Z}\right)$, i.e. $\mathrm{k}\left(\varphi_{2}\right):\left(D, \xi_{D}\right) \rightarrow$ $\left(D^{\prime}, \xi_{D^{\prime}}\right) \in \operatorname{Mod}_{Z}(\mathcal{S})$.

It is easy to see that, for every $\mathrm{E} \in 3-\mathrm{XMod}_{Z}(\mathcal{S})$, we have $G_{3}\left(F_{3}(\mathrm{E})\right) \cong \mathrm{E}$, since $c_{\mathrm{E}} \cdot<0,1>=m_{h_{1}} \cdot\left(1_{X_{0}} \times h_{2}\right) \cdot<0,1>=m_{h_{1}} \cdot<0,1>\cdot h_{2}=$ $b_{2} \cdot h_{2}=\chi_{2}$. For every $\underline{X}_{2, f} \in 2$-Asp $(\mathcal{S} / Z), F_{3}\left(G_{3}\left(\underline{X}_{2, f}\right)\right) \cong \underline{X}_{2, f}$, because $\left(X_{1} \times D, \xi_{X_{1} \times D}\right) \cong\left(X_{2}, \xi_{X_{2}}\right)$. This isomorphism is obtained from the Split Short Five Lemma applied to the commutative diagram

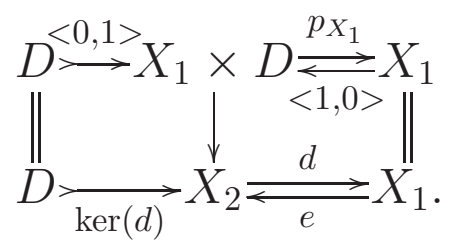

Note that the vertical middle map is $m_{h_{1} \cdot d} \cdot(e \times \operatorname{ker}(\mathrm{k}(d))): X_{1} \times D \rightarrow X_{2}$ and lies in $\operatorname{XMod}(\mathcal{S})$ since it composed by morphisms of $X_{0}$-algebras (Remark 6.8 and Remark 7.2).

For $n \geq 4$, suppose that $k-\operatorname{XMod}_{Z}(\mathcal{S}) \cong(k-1)-\operatorname{Asp}(\mathcal{S} / Z), 2 \leq k \leq n-1$. Using the equivalence when $k=n-1$, an aspherical internal $(n-1)$-groupoid consists of a diagram of the form

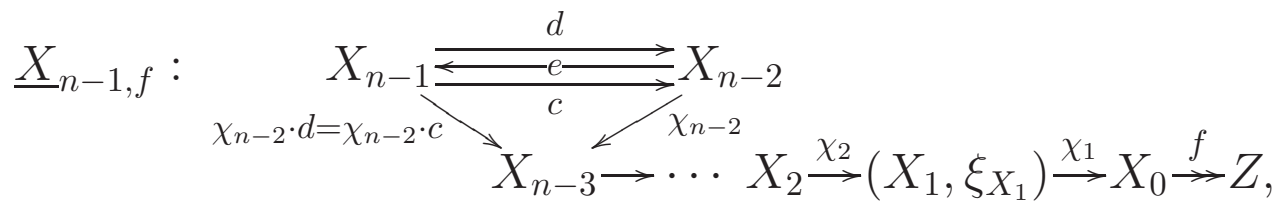

such that the vertical diagrams $\mathrm{k}\left(X_{n-2}\right) \stackrel{\operatorname{ker}\left(\chi_{n-2}\right)}{\longmapsto} X_{n-2} \rightarrow \cdots X_{0} \stackrel{f}{\rightarrow} Z$ and $\mathrm{k}\left(X_{n-1}\right) \stackrel{\operatorname{ker}\left(\chi_{n-2} \cdot d\right)}{\longmapsto} X_{n-1} \rightarrow \cdots X_{0} \stackrel{f}{\rightarrow} Z$ are crossed $(n-1)$-fold extensions, the top line $X_{n-1} \underset{\leftrightarrows}{\leftrightarrows} X_{n-2}$, equipped with a composition morphism $m$, is an 
internal groupoid in $\operatorname{Mod}_{Z}(\mathcal{S})$ and $\left\langle d, c>\right.$ : $X_{n-1} \rightarrow X_{n-2} \times \mathrm{k}\left(X_{n-2}\right)$ is a regular epimorphism (where $\left(p_{X_{n-2}}, m_{X_{n-2}} \cdot\left(1_{X_{n-2}} \times \operatorname{ker}\left(\chi_{n-2}\right)\right): X_{n-2} \times\right.$ $\left.\mathrm{k}\left(X_{n-2}\right) \rightarrow X_{n-2}\right)$ is the kernel pair of $\left.\chi_{n-2}\right)$.

We define $F_{n}: n-\operatorname{XMod}_{Z}(\mathcal{S}) \rightarrow(n-1)-\operatorname{Asp}(\mathcal{S} / Z)$ by

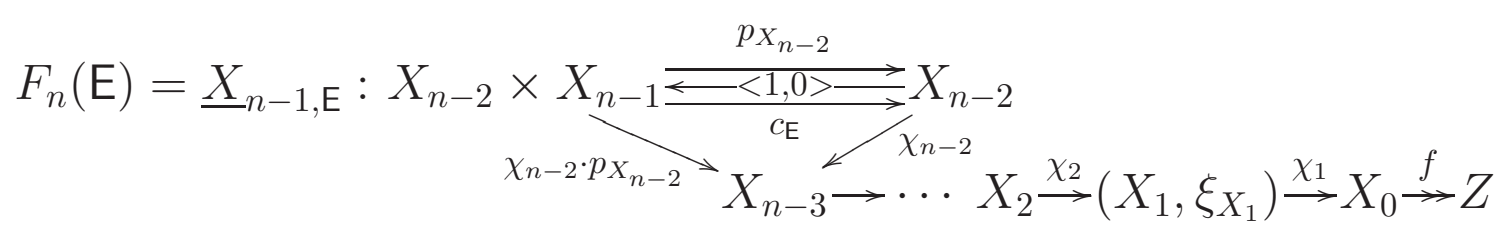

for $\mathrm{E}$ as in (12). Note that:

$$
\begin{aligned}
& \cdot H_{n-1} \stackrel{\operatorname{ker}\left(\chi_{n-2}\right)}{\longmapsto} X_{n-2} \rightarrow \cdots X_{0} \stackrel{f}{\rightarrow} Z, H_{n-1} \times X_{n-1} \stackrel{\operatorname{ker}\left(\chi_{n-2} \cdot p_{X_{n-2}}\right)}{\longmapsto} X_{n-2} \times \\
& X_{n-1} \cdots X_{0} \stackrel{f}{\rightarrow} Z \in(n-1)-\operatorname{XMod}_{Z}(\mathcal{S}) .
\end{aligned}
$$

- $X_{n-2} \times X_{n-1} \underset{c_{\mathrm{E}}}{\stackrel{p_{X_{n-2}}}{\stackrel{<<1,0>}{\longrightarrow}}} X_{n-2}$, with composition $1_{X_{n-2}} \times m_{X_{n-1}}$, is an internal groupoid in $\operatorname{Mod}_{Z}(\mathcal{S})$, for $c_{\mathrm{E}}=m_{X_{n-2}} \cdot\left(1_{X_{n-2}} \times \chi_{n-1}\right)$.

$\cdot<p_{X_{n-2}}, c_{\mathrm{E}}>=1 \times h_{n-1}: X_{n-2} \times X_{n-1} \rightarrow X_{n-2} \times H_{n-1}$ is a regular epimorphism.

For morphisms, we define $F_{n}\left(\alpha, \theta_{n-1}, \cdots, \theta_{2}, \beta, \gamma, 1_{Z}\right)=\left(\theta_{n-1} \times \theta_{n-2}, \theta_{n-2}, \cdots\right.$, $\left.\theta_{2}, \beta, \gamma, 1_{Z}\right)$, which is a morphism in $(n-1)-\operatorname{Asp}(\mathcal{S} / Z)$ since $\left(\theta_{n-1} \times \theta_{n-2}, \theta_{n-2}\right)$ is a morphism of reflexive graphs, $\theta_{n-1}, \cdots, \theta_{2} \in \operatorname{Mod}_{Z}(\mathcal{S})$ and $(\beta, \gamma) \in$ $\operatorname{XMod}(\mathcal{S})$.

We also define $G_{n}:(n-1)-\operatorname{Asp}(\mathcal{S} / Z) \rightarrow n-\operatorname{XMod}_{Z}(\mathcal{S})$ by

$$
G_{n}\left(\underline{X}_{n-1, f}\right)=\mathrm{E}_{\underline{X}_{n-1, f}}: A \stackrel{a}{\longrightarrow} D \stackrel{c \cdot k e r(d)}{\longrightarrow} X_{n-2} \stackrel{\chi_{n-2}}{\longrightarrow} \cdots\left(X_{1}, \xi_{X_{1}}\right) \stackrel{\chi_{1}}{\longrightarrow} X_{0} \stackrel{f}{\longrightarrow} Z
$$

for $\underline{X}_{n-1, f}$ as in (15), $D=\operatorname{ker}(d)$ and $A=\operatorname{ker}(c \cdot \operatorname{ker}(d))$. The proof of the exactness of $\mathrm{E}_{\underline{X}_{n-1, f}}$ is similar to the one made for $n=3$. We already know that $\chi_{1} \in \operatorname{XMod}(\mathcal{S})$ and $X_{2}, \cdots, X_{n-2}, \chi_{2}, \cdots \chi_{n-2}, c \cdot \operatorname{ker}(d) \in$ $\operatorname{Mod}_{Z}(\mathcal{S})$. For morphisms we define $G_{n}\left(\varphi_{n-1}, \varphi_{n-2}, \theta_{n-3}, \cdots, \theta_{2}, \beta, \gamma, 1_{Z}\right)=$ $\left(\mathrm{k}\left(\mathrm{k}\left(\varphi_{n-1}\right)\right), \mathrm{k}\left(\varphi_{n-1}\right), \varphi_{n-2}, \theta_{n-3}, \cdots, \theta_{2}, \beta, \gamma, 1_{Z}\right)$ which is a morphism in $n$ $\operatorname{XMod}_{Z}(\mathcal{S})$ because $(\beta, \gamma) \in \operatorname{XMod}(\mathcal{S})$ and $\theta_{2}, \cdots, \theta_{n-3}, \varphi_{n-2}, \mathrm{k}\left(\varphi_{n-1}\right)$ and $\mathrm{k}\left(\mathrm{k}\left(\varphi_{n-1}\right)\right)$ are in $\in \operatorname{Mod}_{Z}(\mathcal{S})$.

It is easy to see that $F_{n}$ and $G_{n}$ are equivalences (similar to the proof for $F_{3}$ and $\left.G_{3}\right)$.

We use the notations $\underline{X}_{n-1, \mathrm{E}}=F_{n}(\mathrm{E})$ and $\mathrm{E}_{\underline{X}_{n-1, f}}=G_{n}\left(\underline{X}_{n-1, f}\right)$.

Examples 7.4. Based on Example 6.7, the previous equivalence gives us: 
1: $\mathrm{E}_{\nabla_{n-1}(f)}: 1=\cdots 1 \longrightarrow\left(H_{1}, \xi_{H_{1}}\right) \stackrel{b_{1}}{\longrightarrow} X_{0} \stackrel{f}{\longrightarrow} Z$

2: $\mathrm{E}_{\mathrm{K}_{n-1}\left(\mathrm{O}_{A}\right)}: A=A \stackrel{!}{\longrightarrow} 1=\cdots 1 \longrightarrow Z=Z$.

Remark 7.5. Consider $\mathrm{E}_{\underline{X}_{n-1, f}}$ as in (12). The cancelation of level $n-$ 1 gives $\mathrm{E}_{\underline{X}_{n-2, f}}: H_{n-1} \longmapsto X_{n-2} \rightarrow \cdots\left(X_{1}, \xi_{X_{1}}\right) \rightarrow X_{0} \rightarrow Z$. We have: $\mathrm{E}_{\nabla_{n-1}\left(\underline{X}_{n-2, f}\right)}: 1 \longrightarrow H_{n-1} \stackrel{b_{n-1}}{\longrightarrow} X_{n-2} \stackrel{\chi_{n-2}}{\longrightarrow} \cdots\left(X_{1}, \xi_{X_{1}}\right) \stackrel{\chi_{1}}{\longrightarrow} X_{0} \stackrel{f}{\longrightarrow} Z$ and $\mathrm{E}_{\underline{X}_{n-1, f}} \times_{\mathrm{E}_{\nabla_{n-1}\left(\underline{X}_{n-2, f}\right)}} \mathrm{E}_{\underline{X}_{n-1, f}}$ :

$$
A \times A \stackrel{a \times 1}{\longrightarrow} X_{n-1} \times A \stackrel{\chi_{n-1} \cdot p_{X_{n-1}}}{\longrightarrow} X_{n-2} \stackrel{\chi_{n-2}}{\longrightarrow} \cdots\left(X_{1}, \xi_{X_{1}}\right) \stackrel{\chi_{1}}{\longrightarrow} X_{0} \stackrel{f}{\longrightarrow} Z .
$$

7.2. The direction of crossed $n$-fold extensions. The notion of crossed $n$-fold extensions and the equivalence $n-\operatorname{XMod}_{Z}(\mathcal{M}) \cong(n-1)-\operatorname{Asp}(\mathcal{M} / Z)$ establish the link with the direction functor theory of order $n$.

Proposition 7.6. The $n$-th order direction functor $d_{n}: n-\operatorname{XMod}_{Z}(\mathcal{M}) \rightarrow$ $\operatorname{Mod}_{Z}(\mathcal{M})$ assigns to each crossed $n$-fold extension in $\mathcal{M}$ of $A$ by $Z$ a $Z$ module structure on $A$.

Proof: From Proposition 7.3 and the equivalence (6), the $n$-th order direction functor is defined by

$$
\begin{aligned}
d_{n}: n-\mathrm{XMod}_{Z}(\mathcal{M}) & \longrightarrow \operatorname{Mod}_{Z}(\mathcal{M}) . \\
\mathrm{E}_{\underline{X}_{n-1, f}}: A \stackrel{a}{\longrightarrow} X_{n-1} \stackrel{\chi_{n-1}}{\longrightarrow} \cdots\left(X_{1}, \xi_{X_{1}}\right) \stackrel{\chi_{1}}{\longrightarrow} X_{0} \stackrel{f}{\longrightarrow} Z & \longmapsto\left(A, \xi_{\chi_{n-1}}\right)
\end{aligned}
$$

The $Z$-module structure on $A$ is induced by $\chi_{n-1}$ as can be seen by the downward pullback

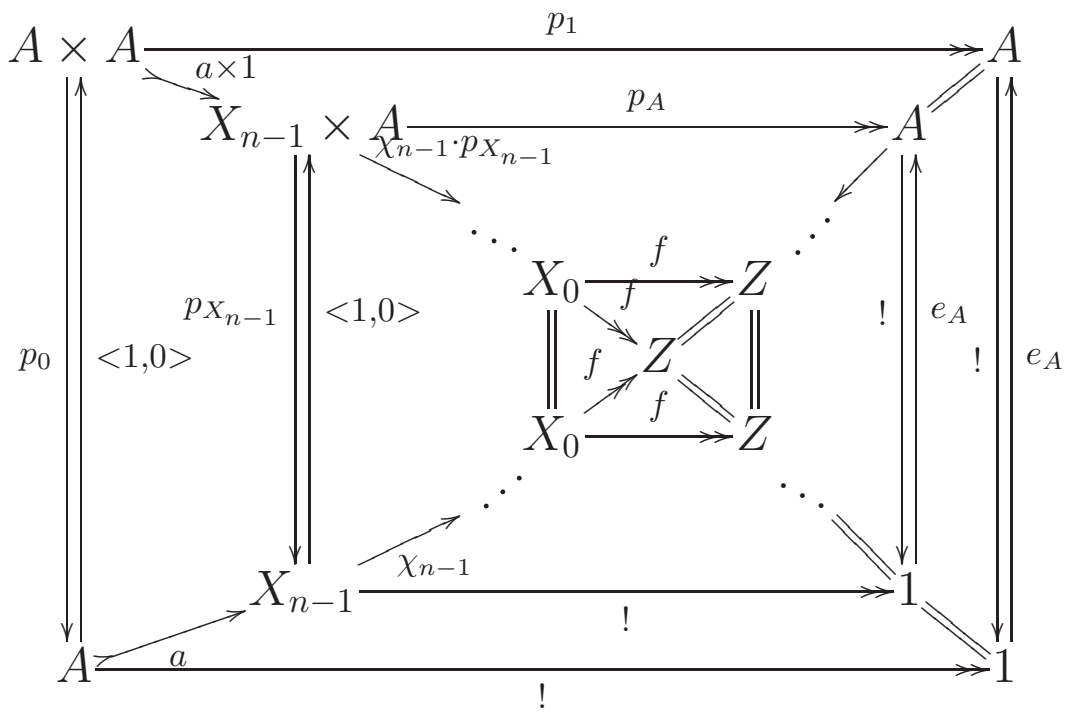


or, equivalently, by the upward pushout, both in $n-\operatorname{XMod}_{Z}(\mathcal{M})$; it corresponds to the diagram of Definition 3.9 for $(n-1)-\operatorname{Asp}(\mathcal{M} / Z)$.

7.3. The Baer sum of crossed $n$-fold extensions, $n \geq 2$. Let $\mathrm{O}_{A}$ be a fixed $Z$-module as in (8). The objects of $d_{n}^{-1}\left(\mathrm{O}_{A}\right)$ are the crossed $n$-fold extensions $\mathrm{E}: A \longmapsto X_{n-1} \stackrel{\chi_{n-1}}{\longrightarrow} \cdots\left(X_{1}, \xi_{X_{1}}\right) \rightarrow X_{0} \rightarrow Z$ in $\mathcal{M}$ of $A$ by $Z$ such that $\mathrm{O}_{\chi_{n-1}} \cong \mathrm{O}_{A}$, i.e. $\left(A, \xi_{\chi_{n-1}}\right) \cong\left(A, \xi_{A}\right)$. We use the classical notation $\operatorname{Opext}^{n}\left(Z, A, \mathrm{O}_{A}\right)$ to represent the abelian group of the equivalence classes of crossed $n$-fold extensions in $d_{n}^{-1}\left(\mathrm{O}_{A}\right)$. The $n$-th cohomology group of the $Z$-module $A$ is (see (4))

$$
\mathbb{H}_{\mathcal{M} / Z}^{n}(A) \cong \pi_{0}\left(d_{n}^{-1}\left(\mathrm{O}_{A}\right)\right)=\operatorname{Opext}^{n}\left(Z, A, \mathrm{O}_{A}\right)
$$

The group operation of $\mathbb{H}_{\mathcal{M} / Z}^{n}(A)$, induced by the tensor product of the monoidal structure of $d_{n}^{-1}\left(\mathrm{O}_{A}\right)$, is called the Baer sum, and defined as [E] + $\left[\mathrm{E}^{\prime}\right]_{\sim}=\left[\mathrm{E} \otimes_{n} \mathrm{E}^{\prime}\right]_{\sim}$. The crossed $n$-fold extension $\mathrm{E} \otimes_{n} \mathrm{E}^{\prime}$ is the codomain of the $d_{n}$-cocartesian morphism $\bar{\mu}_{\mathrm{E}, \mathrm{E}^{\prime}}$ above the multiplication $m_{\mathrm{O}_{A}}$ with domain the product $\mathrm{E} \times{ }_{Z} \mathrm{E}^{\prime}\left(=\left(\mathrm{E} \times \mathrm{E}^{\prime}\right) \Delta_{Z}\right)$ in $n-\mathrm{XMod}_{Z}(\mathcal{M})(\operatorname{Remark} 3.8 .8)$

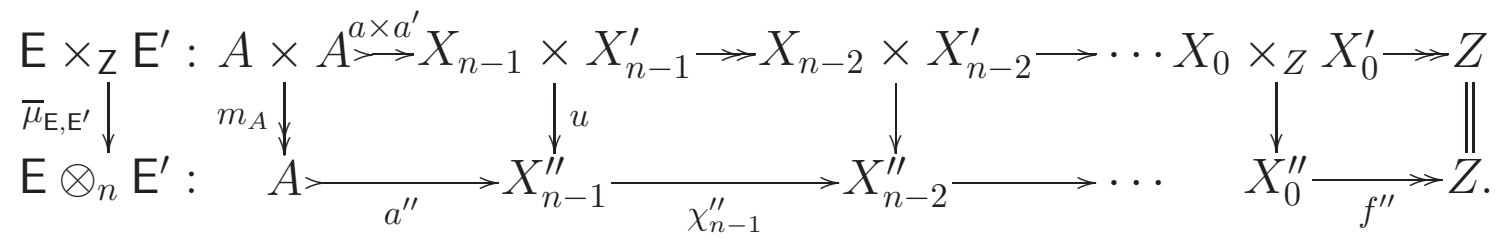

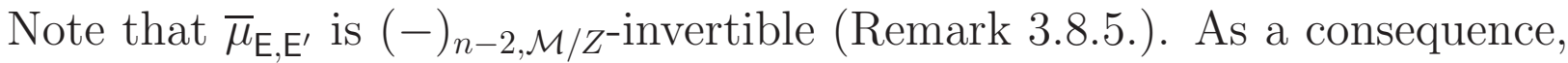
$\mathrm{E} \times{ }_{\mathrm{Z}} \mathrm{E}^{\prime}$ and $\mathrm{E} \otimes_{n} \mathrm{E}^{\prime}$ coincide up to level $n-2$, i.e. all vertical morphism in the diagram above are identities except for $m_{A}$ and $u$. As in Subsection 5.2, $u$ is a regular epimorphism, by Remark 4.9.2., and, by Remark 4.9.1., the left square is a pullback, thus a pushout. So we can conclude that $\mathrm{E} \otimes_{n} \mathrm{E}^{\prime}=$ $m_{A}\left(\mathrm{E} \times \mathrm{E}^{\prime}\right) \Delta_{Z}$, just like the Baer sum of crossed $n$-fold extensions of groups $([20]$ and $[19])$. 
7.4. The long exact sequence. Consider a short exact sequence (9) in $\operatorname{Mod}_{Z}(\mathcal{M})$. The induced long exact sequence is

$$
\begin{aligned}
& 0 \longrightarrow \operatorname{Hom}_{\mathcal{M} / Z}\left(1_{Z}, \rho_{N}\right) \stackrel{k_{*}}{\longrightarrow} \operatorname{Hom}_{\mathcal{M} / Z}\left(1_{Z}, \rho_{P}\right) \stackrel{p_{*}}{\longrightarrow} \operatorname{Hom}_{\mathcal{M} / Z}\left(1_{Z}, \rho_{Q}\right) \stackrel{\delta^{0}}{\longrightarrow} \\
& \longrightarrow \operatorname{Opext}\left(Z, N, \mathrm{O}_{N}\right) \underset{k_{*}^{1}}{\longrightarrow} \operatorname{Opext}\left(Z, P, \mathrm{O}_{P}\right) \underset{p_{*}^{1}}{\longrightarrow} \operatorname{Opext}\left(Z, Q, \mathrm{O}_{Q}\right) \underset{\delta^{1}}{\longrightarrow} \\
& \rightarrow \operatorname{Opext}^{n-1}\left(Z, N, \mathrm{O}_{N}\right)_{*}^{k_{*}^{n-1}} \rightarrow \mathrm{Opext}^{n-1}\left(Z, P, \mathrm{O}_{P}\right) \stackrel{p_{*}^{n-1}}{\rightarrow} \operatorname{Oppext}^{n-1}\left(Z, Q, \mathrm{O}_{Q}\right)^{\delta^{n-1}} \rightarrow \\
& \longrightarrow \operatorname{Opext}^{n}\left(Z, N, \mathrm{O}_{N}\right) \underset{k_{*}^{n}}{\longrightarrow} \operatorname{Oppext}^{n}\left(Z, P, \mathrm{O}_{P}\right) \underset{p_{*}^{n}}{\longrightarrow} \operatorname{Opext}^{n}\left(Z, Q, \mathrm{O}_{Q}\right) \cdots
\end{aligned}
$$

Given $\mathrm{E}_{\underline{X}_{n-2, f}} \in \operatorname{Opext}^{n-1}\left(Z, Q, \mathrm{O}_{Q}\right)$, we define $\delta^{n-1}, n \geq 2$, by (Theorem 2.8)

$$
\begin{aligned}
& \delta^{n-1}\left(\left[\mathrm{E}_{\underline{X}_{n-2, f}}: Q \stackrel{a}{\longrightarrow} X_{n-2} \stackrel{\chi_{n-2}}{\longrightarrow} \cdots\left(X_{1}, \xi_{X_{1}}\right) \stackrel{\chi_{1}}{\longrightarrow} X_{0} \stackrel{f}{\longrightarrow} Z\right]_{\sim}\right)= \\
& =\left[\mathrm{SE}_{\underline{X}_{n-2, f}}: N \stackrel{k}{\longrightarrow} P \stackrel{a \cdot p}{\longrightarrow} X_{n-2} \stackrel{\chi_{n-2}}{\longrightarrow} \cdots\left(X_{1}, \xi_{X_{1}}\right) \stackrel{\chi_{1}}{\longrightarrow} X_{0} \stackrel{f}{\longrightarrow} Z\right]_{\sim} .
\end{aligned}
$$

The crossed $n$-fold extension $\delta^{n-1}\left(\left[\mathrm{E}_{\underline{X}_{n-2, f}}\right]_{\sim}\right)$ is obtained from the pullback

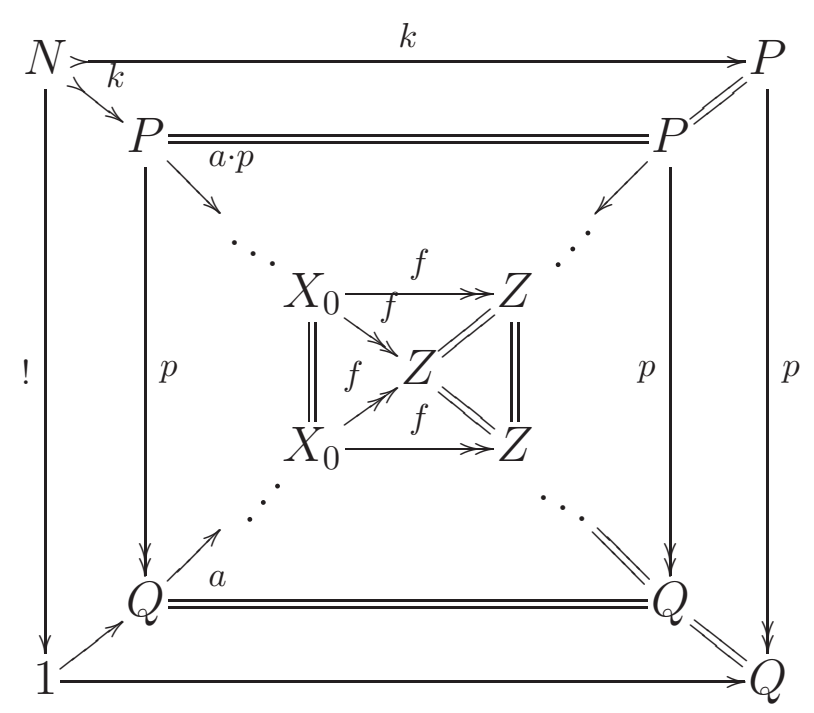

in $n-\mathrm{XMod}_{Z}(\mathcal{M})$. From $d_{n-1}\left(\mathrm{E}_{\underline{X}_{n-2, f}}\right) \cong \mathrm{O}_{Q}$, we obtain a discrete fibration $\mathrm{E}_{\nabla_{n-1}\left(\underline{X}_{n-2, f}\right)} \rightarrow \mathrm{E}_{\mathrm{K}_{n-1}\left(\mathrm{O}_{Q}\right)}$ (Remark 2.4) which is represented by the bottom horizontal morphisms. So, $\delta^{n-1}\left(\left[\mathrm{E}_{\underline{X}_{n-2, f}}\right]_{\sim}\right)$ is given by the Yoneda composition of $\mathrm{S}$ and $\mathrm{E}_{\underline{X}_{n-2, f}}$, just like the definition of the connecting morphism 
for groups ([20] and [19]). Note that, for $n=2, \mathrm{E}_{\underline{X}_{n-2, f}}$ is just a singular extension and the $X_{0}$-algebra structure on $Q$ is $\xi_{Q}^{X_{0}}=\xi_{Q} \cdot \mathrm{k}\left(1_{Q}+f\right)$ and similarly for $P$.

\section{References}

[1] M. Barr, Exact Categories, in: Lecture Notes in Math. 236, Springer (1971), pp. 1-120.

[2] F. Borceux and D. Bourn, Mal'cev, protomodular, homological and semi-abelian categories, Kluwer, 2004.

[3] D. Bourn, The shift functor and the comprehensive factorization for internal groupoids, Cahiers de Topologie et Géometrie Différentielle Categoriques XXVIII (1987) 197-226.

[4] D. Bourn, The tower of $n$-groupoids and the long cohomology sequence, Journal of Pure and Applied Algebra 62 (1989) 137-183.

[5] D. Bourn, Normalization equivalence, kernel equivalence and affine categories, in: Lecture Notes in Math. 1488, Springer (1991), pp. 43-62.

[6] D. Bourn, Baer sums and fibered aspects of Maltsev operations, Cahiers de Topologie et Géometrie Différentielle Categoriques XL (1999) 297-316.

[7] D. Bourn, Normal functors and strong protomodularity, Theory and Applications of Categories 7 (2000) 206-218.

[8] D. Bourn, Normal subobjects and abelian objects in protomodular categories, Journal of Algebra 228 (2000) 143-164.

[9] D. Bourn, $3 \times 3$ lemma and protomodularity, Journal of Algebra 236 (2001), 143-164.

[10] D. Bourn, Aspherical abelian groupoids and their directions, Journal of Pure and Applied Algebra 168 (2002) 133-146.

[11] D. Bourn, Baer sums in homological categories, Journal of Algebra 308 (2007) 414-443.

[12] D. Bourn and G. Janelidze, Protomodularity, descent and semi-direct products, Theory and Applications of Categories 4 (1998) 37-46.

[13] D. Bourn and M. Gran, Centrality and normality in protomodlar categories, Theory and Applications of Categories 9 (2002) 151-165.

[14] D. Bourn and M. Gran, Centrality and connectors in Maltsev categories, Algebra Universalis 48 (2002) 309-331.

[15] D. Bourn and D. Rodelo, Cohomology without projectives, Cahiers de Topologie et Géometrie Différentielle Categoriques XLVIII-2 (2007) 104-153.

[16] J. Duskin, Higher dimensional torsors and the cohomology of topoi: the abelian theory, in: Lecture Notes in Math. 753, Springer (1979), pp. 255-279.

[17] M. Gerstenhaber, A categorical setting for the Baer extension theory, in: Proc. of Symposia in Pure Mathematics 17, pp. 50-64, Amer. Math. Soc., Providence, 1970.

[18] P. Glenn, Realization of cohomology classes by torsors under hypergroupoids, Ph.D. Thesis S.U.N.Y. Buffalo, 1977.

[19] D. Holt, An interpretation of the cohomology groups $H^{n}(G, M)$, J. Algebra, 60, 1979, 307-318.

[20] J. Huebschmann, Crossed $n$-fold extensions of groups and cohomology, Comment. Math. Helv., 55, 1980, 302-314.

[21] G. Janelidze, L. Marki and W. Tholen, Semi-abelian categories, Journal of Pure and Applied Algebra 168 (2002) 367-386.

[22] G. Janelidze, Internal crossed modules, Georgian Mathematical Journal 10 (2003) 99114.

[23] S. Mac Lane, Homology, Springer, 1963. 
[24] D. Rodelo, Moore Categories, Theory and Applications of Categories 12 (2004) 237247.

[25] D. Rodelo, Direç̧ões para a sucessão longa de co-homologia, Thesis, Coimbra, 2005.

DiANA RODELO

Departamento de Matemtica, Universidade do Algarve, 8005-139 Faro, Portugal CMUC, University of Coimbra, 3001-454 Coimbra, Portugal

E-mail address: drodelo@ualg.pt 